\title{
Controlled Lagrangians and the Stabilization of Mechanical Systems I: The First Matching Theorem
}

\author{
Anthony M. Bloch, Member, IEEE, Naomi Ehrich Leonard, Member, IEEE, and Jerrold E. Marsden
}

\begin{abstract}
We develop a method for the stabilization of mechanical systems with symmetry based on the technique of controlled Lagrangians. The procedure involves making structured modifications to the Lagrangian for the uncontrolled system, thereby constructing the controlled Lagrangian. The Euler-Lagrange equations derived from the controlled Lagrangian describe the closed-loop system, where new terms in these equations are identified with control forces. Since the controlled system is Lagrangian by construction, energy methods can be used to find control gains that yield closed-loop stability.

In this paper we use kinetic shaping to preserve symmetry and only stabilize systems modulo the symmetry group. In the sequel to this paper (Part II), we extend the technique to include potential shaping and we achieve stabilization in the full phase space.

The procedure is demonstrated for several underactuated balance problems, including the stabilization of an inverted planar pendulum on a cart moving on a line and an inverted spherical pendulum on a cart moving in the plane.
\end{abstract}

Index Terms-Lagrangian mechanics, nonlinear systems, stabilization.

\section{INTRODUCTION}

$\mathbf{I}$ $\mathrm{N}$ this paper we develop a constructive approach to the derivation of stabilizing control laws for Lagrangian mechanical systems where the Lagrangian has the form of kinetic minus potential energy. The method is Liapunov-based and thus yields large and computable basins of stability, which become asymptotically stable when dissipative controls are added. The methods are designed to be effective for the stabilization of balance systems, such as inverted pendula, as well as for systems with gyroscopic forces such as satellites and underwater vehicles with internal rotors. These examples are worked out in this and companion papers.

The guiding principle behind our methodology is to consider a class of control laws that yield closed-loop dynamics which remain in Lagrangian form. This has the advantage that stabiliza-

Manuscript received September 10, 1998; revised December 20, 1999 and January 13, 2000. Recommended by Associate Editor, O. Egeland. This work was supported in part by the NSF under Grants DMS 9496221 , DMS-9803181, BES 9502477, the AFOSR under Grants F49620-96-1-0100 and F49620-95-1-0419, a Guggenheim Fellowship, Institute for Advanced Study, and by the Office of Naval Research under Grants N00014-96-1-0052 and N00014-98-1-0649.

A. M. Bloch is with the Department of Mathematics, University of Michigan, Ann Arbor, MI 48109 USA (e-mail: abloch@umich.edu).

N. E. Leonard is with the Department of Mechanical and Aerospace Engineering, Princeton University, Princeton, NJ 08544 USA (e-mail: naomi@princeton.edu).

J. E. Marsden is with Control and Dynamical Systems, California Institute of Technology, Pasadena, CA 91125 USA (e-mail: marsden@cds.caltech.edu).

Publisher Item Identifier S 0018-9286(00)10664-6. tion can be understood in terms of energy. In particular, we can make use of energy methods which provide a Liapunov function which gives information on how to choose the control gains to achieve closed-loop stability. Further, even though work is done by the control forces, there is a modification of the mechanical energy of the system that is exactly conserved by the closed-loop dynamics; one can think of it as a combined energy available to the mechanism and the control forces. This can be used to show that, for fixed gains, the control inputs will never need to become large (in time) to achieve stabilization.

Closed-loop dynamics are guaranteed to be Lagrangian by first choosing the closed-loop Lagrangian from a class of controlled Lagrangians that we will explicitly describe. The controlled Lagrangian then provides the control law: the closed-loop dynamics are the Euler-Lagrange equations derived from the controlled Lagrangian and the new terms that appear in the dynamic equations are identified with the control forces. The method ensures that the new terms in the equations of motion only appear in the desired control directions. The associated theory provides sufficient (matching) conditions under which this approach will provide such a control law that yields a closed-loop system in Lagrangian form.

The approach is motivated by a result in [6] for stabilization of unstable middle axis rotation of a rigid spacecraft using a single internal rotor. There, the framework was Hamiltonian and it was shown that the chosen rotor control law was such that the closed-loop system was still Hamiltonian. The new Hamiltonian was a modification of the kinetic energy (Hamiltonian) of the uncontrolled spacecraft. The energy-Casimir method was used to choose the control gain and thereby guarantee closed-loop stability.

The objective of this paper is to demonstrate how the approach of [6] can be generalized and made algorithmic. We switch to a Lagrangian framework from a Hamiltonian framework which helps us to systematize the modification of the uncontrolled Lagrangian to get our controlled Lagrangian. The basic idea behind our approach was introduced in [12] and in [7] and [8]. We remark, however, that there is no reason that our procedure cannot be carried out on the Hamiltonian side. This leads to interesting questions regarding the modified symplectic structures involved and we shall look at this in a future publication. The matching conditions derived in this paper are explicit; for more general, but less explicit conditions, see [2] and [17].

In this paper, we confine ourselves to controlled Lagrangians that only involve modifications to the kinetic energy of the system. Thus, our approach is complementary to that of, for example, [38] and [35]. We can, however, also consider modifications to the potential energy for stabilization and tracking purposes and this is done in the sequel to this paper, Part II [5]. 
The results of the present paper create an energy extremum in the reduced phase space and, accordingly, allow one to deal with stability modulo the symmetries, that is, stability on a reduced phase space. This is clearly a limitation, but this problem is addressed by the introduction of potential shaping, which allows one to achieve stabilization in the full phase space rather than simply modulo the symmetry directions. (For early results in this direction, see [10].) In addition, Part II extends the study of dissipative controllers to the case of potential shaping and, correspondingly, achieves asymptotic stability in the whole phase space, not just the reduced space.

In a forthcoming related paper, [11], we consider the application of our methods to Euler-Poincaré systems which we illustrate with the problems of stabilization of rotation of a rigid spacecraft about its unstable intermediate axis using a single internal rotor and stabilization of the dynamics of an underwater vehicle.

In this paper we restrict ourselves to a class of systems satisfying special matching conditions. This class includes balance systems, such as the inverted planar pendulum on a cart and the inverted spherical pendulum on a cart in the plane, which are mechanically flat, i.e., they lack gyroscopic forces. In a future paper we analyze a more general class of systems which includes examples with gyroscopic forces such as the inverted pendulum on a rotating arm also known as the whirling pendulum (see [9]).

Other relevant work involving energy methods in control and stabilization includes [25], [39], [21], , [22], [3], and [28]. Related ideas on mechanical control systems may also be found in [13], [37], [38], [14], [30], [18], and [19].

Organization of the Paper: In Section I-A, we describe the controlled Lagrangian approach to stabilization. In Section I-B, we apply the approach to stabilization of an inverted pendulum on a cart. In Section II-A we describe the structure of the general class of controlled Lagrangians we consider. In Section II-B we prove the first matching theorem showing that for certain kinds of systems with Abelian symmetry groups and with controls applied to these symmetry directions, one can always find a suitable controlled Lagrangian whose Euler-Lagrange equations give the desired controlled equations. The proof is constructive and shows explicitly how to choose the controlled Lagrangian and identifies the free gain parameters that are needed to achieve stabilization. The control law itself is derived in Section III-A and in Section III-B we give a sufficient condition for closed-loop stability. This is a stabilizability result in the context of the controlled Lagrangian approach and it provides a construction for choosing control gains for stability. In Section IV, we apply the approach to stabilization of inverted pendula, including the case of an inverted spherical pendulum on a cart. In Section $\mathrm{V}$ we show how to modify the control laws to simulate dissipative effects of the right sort to achieve asymptotic stability.

Part II [5], extends the results herein to include potential shaping and tracking. It is shown that one gets asymptotic stability in the full phase space and it deals with such examples as the inverted planar pendulum (and spherical pendulum) on a cart that moves on an incline. Reference [11] proves a matching theorem designed specifically for the case of the Euler-Poincaré equations. This case, not covered by the first matching theorem, is applied to a spacecraft with an internal rotor and to the problem of stabilizing an underwater vehicle using internal rotors. In a further paper we prove a third matching theorem that includes the preceding as two special cases and apply it to a whirling pendulum (motivated by [1]). This is discussed in [9].

\section{A. The Controlled Lagrangian Approach}

In this section we describe in broad terms the mathematics, intuition, and calculational procedure for the method of controlled Lagrangian. This provides a general setting both for the current paper and related papers as described above.

The controlled Lagrangian approach begins with a mechanical system with an uncontrolled (free) Lagrangian equal to kinetic energy minus potential energy. We then modify the kinetic energy (given by a metric tensor) to produce a new controlled Lagrangian which describes the dynamics of the controlled closed-loop system. (As mentioned above the method can be extended to the case of modified potentials and this is described in the forthcoming Part II of this paper.)

The Setting: Suppose our system has configuration space $Q$ and that a Lie group $G$ acts freely and properly on $Q$. It is useful to keep in mind the case in which $Q=S \times G$ with $G$ acting only on the second factor by acting on the left by group multiplication.

For example, for the inverted planar pendulum on a cart (which we consider in detail in Section I-B), $Q=S^{1} \times \mathbb{R}$ with $G=\mathbb{R}$, the group of reals under addition (corresponding to translations of the cart), while for a rigid spacecraft with a rotor (which we treat in a companion paper), $Q=\mathrm{SO}(3) \times S^{1}$, where now the group is $G=S^{1}$, corresponding to rotations of the rotor.

Our goal will be to control the variables lying in the shape space $Q / G$ (in the case in which $Q=S \times G$, then $Q / G=S$ ) using controls which act directly on the variables lying in $G$. We assume that the Lagrangian is invariant under the action of $G$ on $Q$, where the action is on the factor $G$ alone. In many specific examples, such as those given below, the invariance is equivalent to the Lagrangian being cyclic in the $G$-variables. Accordingly, this produces a conservation law for the free system. Our construction will preserve the invariance of the Lagrangian, thus providing us with a controlled conservation law.

The essence of the modification of the Lagrangian involves changing the metric tensor $g(\cdot, \cdot)$ that defines the kinetic energy of the system $(1 / 2) g(\dot{q}, \dot{q})$.

Our method relies on a special decomposition of the tangent spaces to the configuration manifold and a subsequent "controlled" modification of this split. We can describe this as follows.

Horizontal and Vertical Spaces: The tangent space to $Q$ can be split into a sum of horizontal and vertical parts defined as follows: for each tangent vector $v_{q}$ to $Q$ at a point $q \in Q$, we can write a unique decomposition

$$
v_{q}=\operatorname{Hor} v_{q}+\operatorname{Ver} v_{q}
$$

such that the vertical part is tangent to the orbits of the $G$-action and where the horizontal part is the metric orthogonal to the 
vertical space; that is, it is uniquely defined by requiring the identity

$$
g\left(v_{q}, w_{q}\right)=g\left(\operatorname{Hor} v_{q}, \operatorname{Hor} w_{q}\right)+g\left(\operatorname{Ver} v_{q}, \operatorname{Ver} w_{q}\right)
$$

where $v_{q}$ and $w_{q}$ are arbitrary tangent vectors to $Q$ at the point $q \in Q$. This choice of horizontal space coincides with that given by the mechanical connection-see, for example, [31]. One can think intuitively of this decomposition of vectors as a decomposition into a piece in the symmetry, or group direction (the vertical piece) and one in the shape, or internal direction (the horizontal piece). For example, in a vibrating molecule, this would correspond to a decomposition into rotational and vibrational modes. However, it is important to realize that even when $Q=S \times G$, while the vertical space consists of vectors with a zero first component, the horizontal space need not consist of vectors with a zero second component. In examples, deviations from this are important and correspond to the interaction of the dynamics of the shape and group variables.

The Controlled Lagrangian: For the kinetic energy of our controlled Lagrangian, we use a modified version of the right-hand side of (1.2). The potential energy remains unchanged. The modification consists of three ingredients:

1) a different choice of horizontal space denoted $\mathrm{Hor}_{\tau}$;

2) a change $g \rightarrow g_{\sigma}$ of the metric acting on horizontal vectors;

3) a change $g \rightarrow g_{\rho}$ of the metric acting on vertical vectors.

To explain these changes in detail, we will need a little more notation. First of all, we let $\xi_{Q}$ denote the infinitesimal generator corresponding to a Lie algebra element $\xi \in \mathfrak{g}$, where $\mathfrak{g}$ is the Lie algebra of $G$ (see [31] or [32, Ch. 9] for the relevant elementary definitions and properties of Lie groups and group actions). This may be thought of intuitively as infinitesimal group motions of the system. Thus, for each $\xi \in \mathfrak{g}, \xi_{Q}$ is a vector field on the configuration manifold $Q$ and its value at a point $q \in Q$ is denoted $\xi_{Q}(q)$.

Definition 1.1: Let $\tau$ be a Lie-algebra-valued horizontal one form on $Q$; that is, a one form with values in the Lie algebra $\mathfrak{g}$ of $G$ that annihilates vertical vectors. This means that for all vertical vectors $v$, the infinitesimal generator $[\tau(v)]_{Q}$ corresponding to $\tau(v) \in \mathfrak{g}$ is the zero vector field on $Q$. The $\tau$-horizontal space at $q \in Q$ consists of tangent vectors to $Q$ at $q$ of the form $\operatorname{Hor}_{\tau} v_{q}=$ Hor $v_{q}-[\tau(v)]_{Q}(q)$, which also defines $v_{q} \mapsto$ $\operatorname{Hor}_{\tau}\left(v_{q}\right)$, the $\tau$-horizontal projection. The $\tau$-vertical projection operator is defined by $\operatorname{Ver}_{\tau}\left(v_{q}\right):=\operatorname{Ver}\left(v_{q}\right)+[\tau(v)]_{Q}(q)$.

Notice that from these definitions and (1.1), we have

$$
v_{q}=\operatorname{Hor}_{\tau}\left(v_{q}\right)+\operatorname{Ver}_{\tau}\left(v_{q}\right)
$$

just as we did with $\tau$ absent. In fact, this new horizontal subspace can be regarded as defining a new connection, the $\tau$-connection. The horizontal space itself, which by abuse of notation, we also write as just Hor or $\mathrm{Hor}_{\tau}$ of course depends on $\tau$ also, but the vertical space does not-it is the tangent to the group orbit. On the other hand, the projection map $v_{q} \mapsto \operatorname{Ver}_{\tau}\left(v_{q}\right)$ does depend on $\tau$.
Definition 1.2: Given $g_{\sigma}, g_{\rho}$, and $\tau$, we define the controlled Lagrangian to be the following Lagrangian which has the form of a modified kinetic energy minus the potential energy

$$
\begin{aligned}
L_{\tau, \sigma, \rho}(v)=\frac{1}{2}[ & g_{\sigma}\left(\operatorname{Hor}_{\tau} v_{q}, \operatorname{Hor}_{\tau} v_{q}\right) \\
& \left.+g_{\rho}\left(\operatorname{Ver}_{\tau} v_{q}, \operatorname{Ver}_{\tau} v_{q}\right)\right]-V(q)
\end{aligned}
$$

where $V$ is the potential energy.

The equations corresponding to this Lagrangian will be our closed-loop equations. The new terms appearing in those equations corresponding to the directly controlled variables are interpreted as control inputs. The modifications to the Lagrangian are chosen so that no new terms appear in the equations corresponding to the variables that are not directly controlled. We refer to this process as "matching." This matching problem will be studied in detail in subsequent sections.

Another way of expressing what we are doing here is the following. A principal connection on a bundle $Q \rightarrow Q / G$, may be thought of as a Lie-algebra-valued one form and one can obtain a new connection by adding to it a horizontal one form $\tau$. The new horizontal space described in the preceding definition is exactly of this sort.

Special Controlled Lagrangians: In this paper we consider controlled Lagrangians in which we take $g_{\rho}=g$ so that (1.4) describes a controlled Lagrangian of the form $L_{\tau, \sigma}$. In certain examples of interest, including the inverted planar or spherical pendulum on a cart, we not only can choose $g_{\rho}=g$ (i.e., there is no $g_{\rho}$ modification needed), but we can also choose the metric $g_{\sigma}$ to modify the original metric $g$ only in the group directions by a scalar factor $\sigma$. As we shall see in Section II the general formula for the controlled Lagrangian then takes the simplified form

$$
L_{\tau, \sigma}(v)=L\left(v+[\tau(v)]_{Q}(q)\right)+\frac{\sigma}{2} g\left([\tau(v)]_{Q},[\tau(v)]_{Q}\right)
$$

We will develop a formula like this for the more general case of $L_{\tau, \sigma, \rho}(1.4)$ in Section II-A. For the satellite with rotors, for example, and for stabilization in the full phase space one must include the effects of $g_{\rho}$ as well; this modification, consistent with (1.4), is given by formula (2.2). Applications of the general case are discussed in the companion papers mentioned above. We remark in passing that the controlled Lagrangian is a modification of the Kaluza-Klein Lagrangian for a particle in a magnetic field, (see, for example, [32]).

The General Strategy: In outline, the general procedure that one goes through to achieve stabilization is given in the following steps.

1) Start with a mechanical system with a Lagrangian $L$ of the form kinetic minus potential energy and a symmetry group $G$. (In the pendulum-cart example below the symmetry group is translation in the horizontal direction.)

2) Write down the equations of motion for the uncontrolled system.

3) Introduce $\tau, g_{\sigma}$, and $g_{\rho}$ to get the controlled Lagrangian (1.4).

4) Write down the equations of motion corresponding to the controlled Lagrangian and read off the control law $u$ from the equations in the symmetry variables (this will be a conservation law). 


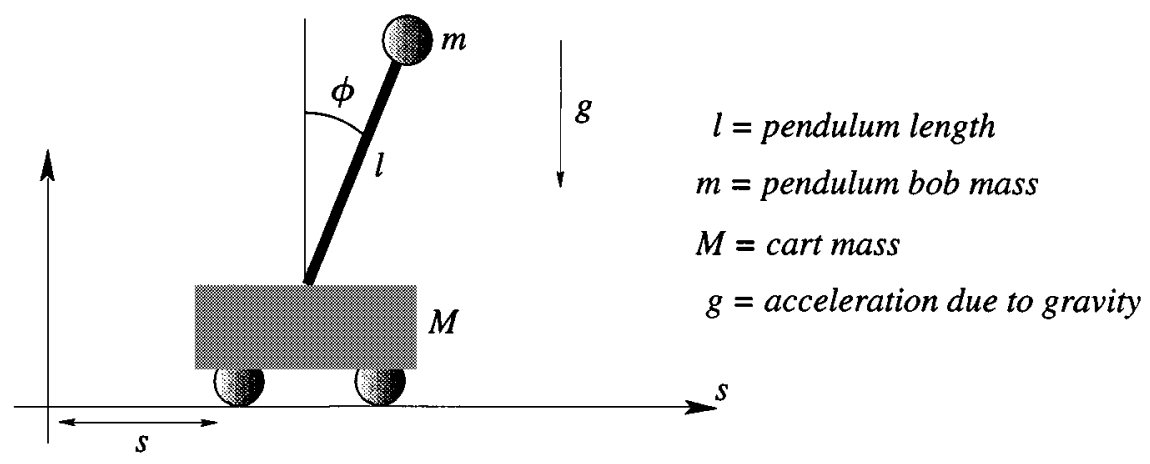

Fig. 1. The pendulum on a cart.

5) Choose $\tau, g_{\sigma}$, and $g_{\rho}$ so that the controlled Euler-Lagrange equations for the original system (i.e., the Euler-Lagrange equations for the Lagrangian $L$ with the control) agree with (that is, match) the Euler-Lagrange equations for the controlled Lagrangian $L_{\tau, \sigma, \rho}$. Determine a feedback law for $u$ by using the Euler-Lagrange equations to eliminate accelerations; then the control law becomes a feedback that is configuration and, possibly, velocity dependent. The general matching theorem can be used to guide these calculations.

6) The stability of an equilibrium is determined by linearization or by the energy-momentum (or, when appropriate, the energy-Casimir-Arnold) method, using any available freedom in the choice of $\tau, g_{\sigma}$, and $g_{\rho}$.

We use this strategy to prove general matching and stabilizability theorems. The matching theorems provide sufficient conditions for successful completion of Steps 1-5 and an explicit construction of the controlled Lagrangian and the control law. In the case that matching is achieved, the stabilizability theorems provide sufficient conditions for closed-loop stability according to Step 6. Again the theory is constructive, providing an explicit choice of control gains for closed-loop stability.

We must emphasize that in doing concrete examples, it can be quite complicated to go through the preceding procedures directly, although we shall do so in the next section for the relatively simple case of the inverted pendulum on a cart. Using the general matching theorems in examples, however, is relatively straightforward.

\section{B. The Inverted Pendulum on a Cart}

Before developing the theory further, we will give an example to show how the ideas work in a concrete setting and to show that the ideas lead to interesting results.

The system we consider is the inverted pendulum on a cart. (The linearized case of this problem was considered in [12].) This example shows the effectiveness of the method for the stabilization of balance systems. Related examples we will treat later are the inverted spherical pendulum on a hockey puck, the satellite with rotors, the underwater vehicle with internal rotors and an inverted pendulum on a rotating arm.

Other examples that we hope will eventually be amenable to these methods include the bicycle (see, for example, [16] and [23]). For these nonholonomic systems, it is hoped that one can use the nonholonomic energy-momentum techniques of [40] to achieve stabilization.

The Lagrangian: First, we set up the Lagrangian for the pendulum-cart system. Let $s$ denote the position of the cart on the $s$-axis and let $\phi$ denote the angle of the pendulum with the upright vertical, as in Fig. 1.

The configuration space for this system is $Q=S \times G=$ $S^{1} \times \mathbb{R}$, with the first factor being the pendulum angle $\phi$ and the second factor being the cart position $s$. The velocity phase space, $T Q$ has coordinates $(\phi, s, \dot{\phi}, \dot{s})$.

The velocity of the cart relative to the lab frame is $\dot{s}$, while the velocity of the pendulum relative to the lab frame is the vector

$$
v_{\text {pend }}=(\dot{s}+l \cos \phi \dot{\phi},-l \sin \phi \dot{\phi}) \text {. }
$$

The system kinetic energy is the sum of the kinetic energies of the cart and the pendulum

$$
K(\phi, s, \dot{\phi}, \dot{s})=\frac{1}{2}[\dot{\phi}, \dot{s}]\left[\begin{array}{cc}
m l^{2} & m l \cos \phi \\
m l \cos \phi & M+m
\end{array}\right]\left[\begin{array}{c}
\dot{\phi} \\
\dot{s}
\end{array}\right] .
$$

The Lagrangian is the kinetic minus potential energy, so we get

$$
L(\phi, s, \dot{\phi}, \dot{s})=K(\phi, s, \dot{\phi}, \dot{s})-V(\phi)
$$

where the potential energy is $V=m g l \cos \phi$.

The symmetry group $G$ of the pendulum-cart system is that of translation in the $s$ variable, so $G=\mathbb{R}$. We do not destroy this symmetry when doing stabilization in $\phi$; we would, however, use symmetry-breaking potentials to track in the variable $s$ if tracking were our goal. In this paper we are focusing on stabilizing this and similar balance systems.

For notational convenience we rewrite the Lagrangian as

$$
L(\phi, s, \dot{\phi}, \dot{s})=\frac{1}{2}\left(\alpha \dot{\phi}^{2}+2 \beta \cos \phi \dot{s} \dot{\phi}+\gamma \dot{s}^{2}\right)+D \cos \phi
$$

where $\alpha=m l^{2}, \beta=m l, \gamma=M+m$, and $D=-m g l$ are constants. Note that $\alpha \gamma-\beta^{2}>0$, reflecting the positive definiteness of the mass matrix (i.e., the metric). The momentum conjugate to $\phi$ is

$$
p_{\phi}=\frac{\partial L}{\partial \dot{\phi}}=\alpha \dot{\phi}+\beta \cos \phi \dot{s}
$$

and the momentum conjugate to $s$ is

$$
p_{s}=\frac{\partial L}{\partial \dot{s}}=\gamma \dot{s}+\beta \cos \phi \dot{\phi} .
$$

The relative equilibrium defined by $\phi=0, \dot{\phi}=0$, and $\dot{s}=0$ is unstable since $D<0$. 
Equations of Motion: The equations of motion for the pendulum-cart system with a control force $u$ acting on the cart (and no direct forces acting on the pendulum) are, since $s$ is a cyclic variable

$$
\begin{aligned}
\frac{d}{d t} \frac{\partial L}{\partial \dot{\phi}}-\frac{\partial L}{\partial \phi} & =0 \\
\frac{d}{d t} \frac{\partial L}{\partial \dot{s}} & =u
\end{aligned}
$$

i.e.,

$$
\frac{d}{d t} p_{\phi}+\beta \sin \phi \dot{s} \dot{\phi}+D \sin \phi=0
$$

that is

and

$$
\frac{d}{d t}(\alpha \dot{\phi}+\beta \cos \phi \dot{s})+\beta \sin \phi \dot{s} \dot{\phi}+D \sin \phi=0
$$

$$
\frac{d}{d t} p_{s}=\frac{d}{d t}(\gamma \dot{s}+\beta \cos \phi \dot{\phi})=u .
$$

The Controlled Lagrangian: Next, we form the controlled Lagrangian by modifying only the kinetic energy of the free pendulum-cart system according to the procedure given in the preceding section. This involves a nontrivial choice of $\tau$ and $g_{\sigma}$, but in this case, as we have remarked, it is sufficient to let $g_{\rho}=g$.

The most general $s$-invariant horizontal one form $\tau$ is given by $\tau=k(\phi) d \phi$ and we choose $g_{\sigma}$ to modify $g$ in the group direction by a constant scalar factor $\sigma$ (in general, $\sigma$ need not be a constant, but it is for the present class of examples). Using (1.5), we let

$$
\begin{aligned}
L_{\tau, \sigma}:= & \frac{1}{2}\left(\alpha \dot{\phi}^{2}+2 \beta \cos \phi(\dot{s}+k \dot{\phi}) \dot{\phi}+\gamma(\dot{s}+k \dot{\phi})^{2}\right) \\
& +\frac{\sigma}{2} \gamma k^{2} \dot{\phi}^{2}+D \cos \phi
\end{aligned}
$$

Notice that the variable $s$ is still cyclic. Following the guidelines of the theory, we look for the feedback control by looking at the change in the conservation law. Associated to the new Lagrangian $L_{\tau, \sigma}$, we have the conservation law

$$
\frac{d}{d t}\left(\frac{\partial L_{\tau, \sigma}}{\partial \dot{s}}\right)=\frac{d}{d t}(\beta \cos \phi \dot{\phi}+\gamma(\dot{s}+k \dot{\phi}))=0
$$

which we can rewrite in terms of the conjugate momentum $p_{s}$ for the uncontrolled Lagrangian as

$$
\frac{d}{d t} p_{s}=u:=-\frac{d}{d t}(\gamma k(\phi) \dot{\phi}) \text {. }
$$

Thus, we identify the term on the right hand side with the control force exerted on the cart.

Using the controlled Lagrangian and (1.12), the $\phi$ equation is computed to be

$$
\begin{aligned}
& \left(\alpha-\frac{\beta^{2}}{\gamma} \cos ^{2} \phi+\sigma \gamma k^{2}(\phi)\right) \ddot{\phi} \\
& \quad+\left(\frac{\beta^{2}}{\gamma} \cos \phi \sin \phi+\sigma \gamma k(\phi) k^{\prime}(\phi)\right) \dot{\phi}^{2} \\
& \quad+D \sin \phi=0 .
\end{aligned}
$$

Matching: The next step is to make choices of $k$ and $\sigma$ so that (1.14) using the controlled Lagrangian agrees with the $\phi$ equation for the controlled cart (1.10) with the control law given by (1.13). The $\phi$ equation for the controlled cart is

$$
\begin{aligned}
& \left(\alpha-\frac{\beta^{2}}{\gamma} \cos ^{2} \phi-\beta k(\phi) \cos \phi\right) \ddot{\phi} \\
& \quad+\left(\frac{\beta^{2}}{\gamma} \cos \phi \sin \phi-\beta \cos \phi k^{\prime}(\phi)\right) \dot{\phi}^{2} \\
& \quad+D \sin \phi=0 .
\end{aligned}
$$

Comparing (1.14) and (1.15) we see that we require (twice)

$$
\sigma \gamma[k(\phi)]^{2}=-\beta k(\phi) \cos \phi .
$$

Since $\sigma$ was assumed to be a constant we set

$$
k(\phi)=\kappa \frac{\beta}{\gamma} \cos \phi
$$

where $\kappa$ is a dimensionless constant (so $\sigma=-1 / \kappa$ ).

The Control Law: Substituting for $\ddot{\phi}$ and $k$ in (1.13) we obtain the desired nonlinear control law

$$
u=\frac{\kappa \beta \sin \phi\left(\alpha \dot{\phi}^{2}+\cos \phi D\right)}{\alpha-\frac{\beta^{2}}{\gamma}(1+\kappa) \cos ^{2} \phi} .
$$

Stabilization: By examining either the energy or the linearization of the closed-loop system, one can see that the equilibrium $\phi=\dot{\phi}=\dot{s}=0$ is stable if

$$
\kappa>\frac{\alpha \gamma-\beta^{2}}{\beta^{2}}=\frac{M}{m}>0 .
$$

In summary, we get a stabilizing feedback control law for the inverted pendulum provided $\kappa$ satisfies (1.19). As mentioned in the introduction, this means stability in the reduced space, that is, modulo translations. Concretely, this means that one has stability in the pendulum position, but not in the cart position, even though, as we shall see, with dissipation, one can bring the cart velocity to zero. Our work on potential shaping (Part II; see also [10]) demonstrates how to obtain stability in the cart position also.

A calculation shows that the denominator of $u$ is nonzero for $\phi$ satisfying $\sin ^{2} \phi<E / F$ where $E=\kappa-\left(\alpha \gamma-\beta^{2}\right) / \beta^{2}(E$ is positive if the stability condition holds) and $F=\kappa+1$. The range of $\phi$ tends to the range $-\pi / 2<\phi<\pi / 2$ for large $\kappa$.

The above remark suggests that the region of stability (or attraction when damping control is added) is the whole range of nondownward pointing states. In fact, we assert that this method produces large computable domains of attraction for stabilization.

This approach has advantages because it is done within the context of mechanics; one can understand the stabilization in terms of the effective creation of an inverted energy well by the feedback control. (Our feedback in general creates a maximum for balance systems, since for these systems the equilibrium is a maximum of the potential energy which we do not modify.) As discussed in Section V, the system is then robustly stabilized by the addition of appropriate dissipation. Note also that the linearized feedback is just proportional feedback.

Remark: The matching procedure does not involve the actual value of the new conserved quantity for the controlled 
system - this is also true for all three of the matching theorems in this paper. The value of the conserved quantity was used in [6] because a symmetry reduction was performed.

\section{THE First MATCHING THEOREM}

In this section, we prove the first of three major matching theorems: the case of $g_{\rho}=g$.

The main goal is to abstract what was happening for the case of the inverted planar pendulum and prove a general matching theorem that applies to such examples. We apply the matching result to the more sophisticated case of the inverted spherical pendulum in Section IV-B.

As shown in companion papers, more general results (e.g., where $g_{\rho} \neq g$ ) are needed for the case of the satellite and the underwater vehicle as well as the whirling pendulum.

\section{A. The Structure of $L_{\tau, \sigma, \rho}$}

As we have mentioned, while we needed only $L_{\tau, \sigma}$ for the inverted pendulum, we will eventually need $L_{\tau, \sigma, \rho}$ for the satellite with a rotor, the underwater vehicle, and the inverted pendulum on a rotor arm.

In this section we prove a structure theorem for $L_{\tau, \sigma, \rho}$ that proves and generalizes (1.5). Recall that this formula was already helpful in the case of the inverted pendulum; likewise, the formula below will be useful in our first matching theorem and in the case of the satellite and the underwater vehicle, etc.

We begin by recalling the definition of the controlled Lagrangian

$$
\begin{aligned}
L_{\tau, \sigma, \rho}(v)=\frac{1}{2}[ & g_{\sigma}\left(\operatorname{Hor}_{\tau} v_{q}, \operatorname{Hor}_{\tau} v_{q}\right) \\
& \left.+g_{\rho}\left(\operatorname{Ver}_{\tau} v_{q}, \operatorname{Ver}_{\tau} v_{q}\right)\right]-V(q)
\end{aligned}
$$

and we make the following assumptions on the metric $g_{\sigma}$ (these assumptions are also appropriate for the case of $L_{\tau, \sigma}$ that we considered earlier).

1) $g=g_{\sigma}$ on Hor.

2) Hor and Ver are orthogonal for $g_{\sigma}$.

Keep in mind that Hor denotes the horizontal space for the given uncontrolled system and that $\mathrm{Hor}_{\tau}$ denotes the horizontal space as modified by the one form $\tau$. Note also that the new metrics $g_{\sigma}$ and $g_{\rho}$ will modify $g$ on Ver, the vertical space (or group directions), which is independent of any modification due to $\tau$. On the other hand, also recall that the vertical projection operator

$$
\operatorname{Ver}_{\tau}\left(v_{q}\right):=\operatorname{Ver}\left(v_{q}\right)+[\tau(v)]_{Q}(q)
$$

does depend on $\tau$.

Theorem 2.1: We have the following formula:

$$
L_{\tau, \sigma, \rho}(v)=L\left(v+\tau(v)_{Q}\right)+\frac{1}{2} g_{\sigma}\left(\tau(v)_{Q}, \tau(v)_{Q}\right)+\frac{1}{2} \varpi(v)
$$

where $v \in T_{q} Q$ and where $\varpi(v)=\left(g_{\rho}-g\right)\left(\operatorname{Ver}_{\tau}(v), \operatorname{Ver}_{\tau}(v)\right)$. Note that if $g_{\rho}=g$ (so that $\varpi=0$ ) and if $g_{\sigma}$ is a scalar times $g$ in the group directions, then this formula reduces to (1.5).
Proof: We begin by manipulating the first ("kinetic energy") term of $L_{\tau, \sigma, \rho},(2.1)$, using the given properties of $g_{\sigma}$ and the definition of the $\tau$-horizontal operator $\operatorname{Hor}_{\tau}\left(v_{q}\right)=\operatorname{Hor}\left(v_{q}\right)-\tau\left(v_{q}\right)_{Q}$

$$
\begin{aligned}
& \frac{1}{2}\left[g_{\sigma}\left(\operatorname{Hor}_{\tau} v_{q}, \operatorname{Hor}_{\tau} v_{q}\right)\right] \\
& \quad=\frac{1}{2}\left[g_{\sigma}\left(\operatorname{Hor}(v)-\tau(v)_{Q}, \operatorname{Hor}(v)-\tau(v)_{Q}\right)\right] \\
& \quad=\frac{1}{2}\left[g(\operatorname{Hor}(v), \operatorname{Hor}(v))+g_{\sigma}\left(\tau(v)_{Q}, \tau(v)_{Q}\right)\right] .
\end{aligned}
$$

Write the second term of (2.1) as

$$
\frac{1}{2}\left[g_{\rho}\left(\operatorname{Ver}_{\tau} v_{q}, \operatorname{Ver}_{\tau} v_{q}\right)\right]=\frac{1}{2}\left[g\left(\operatorname{Ver}_{\tau} v_{q}, \operatorname{Ver}_{\tau} v_{q}\right)+\varpi(v)\right] .
$$

Now write

$$
\begin{aligned}
\frac{1}{2}[ & \left.g\left(\operatorname{Ver}_{\tau} v_{q}, \operatorname{Ver}_{\tau} v_{q}\right)\right] \\
= & \frac{1}{2}\left[g\left(\operatorname{Ver}(v)+\tau(v)_{Q}, \operatorname{Ver}(v)+\tau(v)_{Q}\right)\right] \\
= & \frac{1}{2} g(\operatorname{Ver}(v), \operatorname{Ver}(v))+g\left(\operatorname{Ver}(v), \tau(v)_{Q}\right) \\
& +\frac{1}{2} g\left(\tau(v)_{Q}, \tau(v)_{Q}\right) \\
= & \frac{1}{2} g(\operatorname{Ver}(v), \operatorname{Ver}(v))+g\left(v, \tau(v)_{Q}\right) \\
& +\frac{1}{2} g\left(\tau(v)_{Q}, \tau(v)_{Q}\right)
\end{aligned}
$$

since $v=\operatorname{Hor}(v)+\operatorname{Ver}(v)$ and the horizontal space is $g$-orthogonal to the vertical space. Substituting this last expression into the second term of (2.1) and adding it to the first term gives

$$
\begin{aligned}
& \frac{1}{2} g(v, v)+g\left(v, \tau(v)_{Q}\right)+\frac{1}{2} g\left(\tau(v)_{Q}, \tau(v)_{Q}\right) \\
& \quad+\frac{1}{2} g_{\sigma}\left(\tau(v)_{Q}, \tau(v)_{Q}\right)+\frac{1}{2} \varpi(v)
\end{aligned}
$$

which equals

$$
\frac{1}{2} g\left(v+\tau(v)_{Q}, v+\tau(v)_{Q}\right)+\frac{1}{2} g_{\sigma}\left(\tau(v)_{Q}, \tau(v)_{Q}\right)+\frac{1}{2} \varpi(v) .
$$

Subtracting the potential gives the desired expression.

\section{B. The First Matching Theorem}

Introduction: Motivated by the inverted planar pendulum on a cart, in this section we prove the first matching theorem for mechanical systems such as the inverted pendulum for which we can take $g_{\rho}=g$. The group $G$ associated with the control directions will be assumed to be Abelian. We illustrate this case in Section IV with inverted pendula, including the inverted spherical pendulum on a two-dimensional "cart."

Roughly speaking, the class of systems covered by the first matching theorem are those whose control forces are in the direction of an Abelian symmetry group (such as the translation direction for the pendulum on a cart), whose inertial properties are independent of the internal configuration of the system (such as the total translational inertia of the cart pendulum system is independent of the angle of the pendulum) and whose gyroscopic structure satisfies a certain symmetry condition. The exact hypotheses are spelled out in Assumptions M-1-M-3 discussed hereunder.

All of the matching theorems are constructive; they show explicitly how to pick the controlled Lagrangian to achieve the desired matching in a way that generalizes the example of the inverted planar pendulum on a cart.

The Controlled Lagrangian Identity: Let Hor be the horizontal space for the given kinetic energy metric as explained 
earlier, let $\tau$ be a horizontal one form, and let $\mathrm{Hor}_{\tau}$ be the new horizontal space as explained earlier. Define $L_{\tau, \sigma, \rho}$ according to Definition 1.2. Theorem 2.1 for our controlled Lagrangian says that

$$
L_{\tau, \sigma, \rho}(v)=L\left(v+\tau(v)_{Q}\right)+\frac{1}{2} g_{\sigma}\left(\tau(v)_{Q}, \tau(v)_{Q}\right)+\frac{1}{2} \varpi(v)
$$

where $\varpi=g_{\rho}-g$ on the vertical space. For this section, we choose $g_{\rho}=g$, so $\varpi=0$ and we get

$$
L_{\tau, \sigma}(v)=L\left(v+\tau(v)_{Q}\right)+\frac{1}{2} g_{\sigma}\left(\tau(v)_{Q}, \tau(v)_{Q}\right)
$$

This formula will be extremely useful for the first matching theorem, which we shall perform using a coordinate calculation.

Notation: Locally, we write coordinates for $Q$ as $x^{\alpha}, \theta^{a}$ where $x^{\alpha}, \alpha=1, \ldots n$ are coordinates on the shape space $Q / G$ and where $\theta^{a}, a=1, \ldots, r$ are coordinates for the Abelian group $G$. For the uncontrolled system, the variables $\theta^{a}$ will be cyclic coordinates in the classical sense. We write the given Lagrangian in these coordinates (with the summation convention in force) as

$$
\begin{aligned}
& L\left(x^{\alpha}, \dot{x}^{\beta}, \dot{\theta}^{a}\right) \\
& \quad=\frac{1}{2} g_{\alpha \beta} \dot{x}^{\alpha} \dot{x}^{\beta}+g_{\alpha a} \dot{x}^{\alpha} \dot{\theta}^{a}+\frac{1}{2} g_{a b} \dot{\theta}^{a} \dot{\theta}^{b}-V\left(x^{\alpha}\right) .
\end{aligned}
$$

The Conserved Quantity: The conserved quantity, that is, the momentum conjugate to the cyclic variable $\theta^{a}$ for the preceding Lagrangian, is given by

$$
J_{a}=\frac{\partial L}{\partial \dot{\theta}^{a}}=g_{\alpha a} \dot{x}^{\alpha}+g_{a b} \dot{\theta}^{b} .
$$

The Controlled Euler-Lagrange Equations: The equations of motion for the control system where the controls $u_{a}$ act in the $\theta^{a}$ directions are the controlled Euler-Lagrange equations

$$
\begin{aligned}
\frac{d}{d t} \frac{\partial L}{\partial \dot{x}^{\alpha}}-\frac{\partial L}{\partial x^{\alpha}} & =0 \\
\frac{d}{d t} \frac{\partial L}{\partial \dot{\theta}^{a}} & =u_{a} .
\end{aligned}
$$

Coordinate Formulas for the Horizontal and Vertical Projections: We now embark on the development of coordinate formulas for the controlled Lagrangian. To do this, we first develop coordinate formulas for the horizontal and vertical projections.

For a vector $v=\left(\dot{x}^{\alpha}, \dot{\theta}^{a}\right)$, and suppressing the base point $\left(x^{\alpha}, \theta^{a}\right)$ in the notation, its horizontal and vertical projections are verified to be

$$
\begin{aligned}
& \operatorname{Hor}(v)=\left(\dot{x}^{\alpha},-g^{a b} g_{\alpha b} \dot{x}^{\alpha}\right) \\
& \operatorname{Ver}(v)=\left(0, \dot{\theta}^{a}+g^{a b} g_{\alpha b} \dot{x}^{\alpha}\right)
\end{aligned}
$$

where, as is standard practice, $g^{a b}$ denotes the inverse of the matrix $g_{a b}$. Notice that $v=\operatorname{Hor}(v)+\operatorname{Ver}(v)$, as it should.

These formulas can also be obtained systematically using the formulas for the mechanical connection in terms of the locked inertia tensor, as in, for example, [31]. (In the present context, the locked inertia tensor is the tensor $\mathbb{I}_{a b}=g_{a b}$.)
The $\tau$-Horizontal and Vertical Projections: We shall write the given horizontal one form $\tau$ in coordinates as $\tau^{a}=\tau_{\alpha}^{a} d x^{\alpha}$. Thus

$$
[\tau(v)]_{Q}=\left(0, \tau_{\alpha}^{a} \dot{x}^{\alpha}\right) .
$$

The corresponding $\tau$-horizontal and vertical operators are checked to be

$$
\begin{aligned}
& \operatorname{Hor}_{\tau}(v)=\left(\dot{x}^{\alpha},-g^{a b} g_{\alpha b} \dot{x}^{\alpha}-\tau_{\alpha}^{a} \dot{x}^{\alpha}\right) \\
& \operatorname{Ver}_{\tau}(v)=\left(0, \dot{\theta}^{a}+g^{a b} g_{\alpha b} \dot{x}^{\alpha}+\tau_{\alpha}^{a} \dot{x}^{\alpha}\right) .
\end{aligned}
$$

Coordinate Formula for $L_{\tau, \sigma}$ : We shall first develop a useful coordinate formula for $L_{\tau, \sigma}$. We write down the coordinate form of the definition followed by the coordinate form of the identity given in Theorem 2.1 .

First of all, we write down the coordinate formula for $L_{\tau, \sigma}$ using the definition, namely (2.1) with $g_{\rho}=g$, along with the preceding coordinate formulas for the horizontal and vertical projections to get

$$
\begin{aligned}
L_{\tau, \sigma}= & \frac{1}{2} \sigma_{\alpha \beta} \dot{x}^{\alpha} \dot{x}^{\beta}+\sigma_{a \beta} \dot{x}^{\beta}\left(-g^{a b} g_{\alpha b} \dot{x}^{\alpha}-\tau_{\alpha}^{a} \dot{x}^{\alpha}\right) \\
& +\frac{1}{2} \sigma_{a b}\left(g^{a c} g_{\alpha c} \dot{x}^{\alpha}+\tau_{\alpha}^{a} \dot{x}^{\alpha}\right)\left(g^{b d} g_{\beta d} \dot{x}^{\beta}+\tau_{\beta}^{b} \dot{x}^{\beta}\right) \\
& +\frac{1}{2} g_{a b}\left(\dot{\theta}^{a}+g^{a c} g_{\alpha c} \dot{x}^{\alpha}+\tau_{\alpha}^{a} \dot{x}^{\alpha}\right) \\
& \times\left(\dot{\theta}^{b}+g^{b d} g_{\beta d} \dot{x}^{\beta}+\tau_{\beta}^{b} \dot{x}^{\beta}\right)-V .
\end{aligned}
$$

Remark on Notation: We use the notation $\sigma_{a b}$ for the $a b$ components of $g_{\sigma}$ and, later on, shall likewise use notation $\rho_{a b}$ for the $a b$ components of $g_{\rho}$.

Returning to the preceding calculation, (2.3) gives

$$
L_{\tau, \sigma}=L\left(x^{\alpha}, \dot{x}^{\beta}, \dot{\theta}^{a}+\tau_{\alpha}^{a} \dot{x}^{\alpha}\right)+\frac{1}{2} \sigma_{a b} \tau_{\alpha}^{a} \tau_{\beta}^{b} \dot{x}^{\alpha} \dot{x}^{\beta} .
$$

The equivalence of these two formulas may also be checked by a direct calculation in this case.

The Controlled Conserved Quantity: From (2.10) or (2.11), and (2.4), we find that the associated controlled conserved quantity is given by

$$
\begin{aligned}
\tilde{J}_{a} & =\frac{\partial L_{\tau, \sigma}}{\partial \dot{\theta}^{a}}=\frac{\partial L}{\partial \dot{\theta}^{a}}\left(x^{\alpha}, \dot{x}^{\alpha}, \dot{\theta}^{b}+\tau_{\alpha}^{b} \dot{x}^{\alpha}\right) \\
& =g_{\alpha a} \dot{x}^{\alpha}+g_{a b}\left(\dot{\theta}^{b}+\tau_{\alpha}^{b} \dot{x}^{\alpha}\right) .
\end{aligned}
$$

We can also write this as

$$
\tilde{J}_{a}=J_{a}+g_{a b} \tau_{\alpha}^{b} \dot{x}^{\alpha}
$$

Matching Euler-Lagrange Expressions: The $\theta^{a}$-Euler-Lagrange equations for the controlled Lagrangian, which are equivalent to the controlled conservation law, will be used in Section III-B to determine the control law, consistent with the fact that this is the direction in which we are assuming we have control actuation. 
Thus, our first job is to make sure that the $x^{\alpha}$-Euler-Lagrange equations for $L$ and for $L_{\tau, \sigma}$ agree. To do this, we let

$$
\mathcal{E}_{x}\left(L_{\tau, \sigma}\right)=\frac{d}{d t} \frac{\partial L_{\tau, \sigma}}{\partial \dot{x}^{\alpha}}-\frac{\partial L_{\tau, \sigma}}{\partial x^{\alpha}}
$$

denote the $x^{\alpha}$-component of the Euler-Lagrange expression for our controlled Lagrangian $L_{\tau, \sigma}$.

Assume that the Euler-Lagrange equations for $L$ hold. We want to see under what matching conditions they also hold for $L_{\tau, \sigma}$. From (2.11), and subtracting the Euler-Lagrange expression for $L$ (this expression is zero by assumption) from that for $L_{\tau, \sigma}$, we have

$$
\begin{aligned}
\mathcal{E}_{x}\left(L_{\tau, \sigma}\right) & \\
= & \frac{d}{d t}\left[\frac{\partial L}{\partial \dot{x}^{\alpha}}\left(x^{\delta}, \dot{x}^{\delta}, \dot{\theta}^{a}+\tau_{\beta}^{a} \dot{x}^{\beta}\right)-\frac{\partial L}{\partial \dot{x}^{\alpha}}\left(x^{\delta}, \dot{x}^{\delta}, \dot{\theta}^{a}\right)\right] \\
& -\left[\frac{\partial L}{\partial x^{\alpha}}\left(x^{\delta}, \dot{x}^{\delta}, \dot{\theta}^{a}+\tau_{\beta}^{a} \dot{x}^{\beta}\right)-\frac{\partial L}{\partial x^{\alpha}}\left(x^{\delta}, \dot{x}^{\delta}, \dot{\theta}^{a}\right)\right. \\
& \left.+\frac{\partial L}{\partial \dot{\theta}^{b}}\left(x^{\delta}, \dot{x}^{\delta}, \dot{\theta}^{a}+\tau_{\beta}^{a} \dot{x}^{\beta}\right) \tau_{\delta, \alpha}^{b} \dot{x}^{\delta}\right] \\
& +\frac{d}{d t}\left[\frac{\partial L}{\partial \dot{\theta}^{b}}\left(x^{\delta}, \dot{x}^{\delta}, \dot{\theta}^{a}+\tau_{\beta}^{a} \dot{x}^{\beta}\right) \tau_{\alpha}^{b}+\sigma_{a c} \tau_{\beta}^{a} \tau_{\alpha}^{c} \dot{x}^{\beta}\right] \\
& -\frac{\partial}{\partial x^{\alpha}}\left[\frac{1}{2} \sigma_{a b} \tau_{\delta}^{a} \tau_{\beta}^{b} \dot{x}^{\delta} \dot{x}^{\beta}\right]
\end{aligned}
$$

in which the partial derivatives with respect to $L$ denote slot derivatives, where summation over repeated indexes is understood and where $\tau_{\delta, \alpha}^{b}=\partial \tau_{\delta}^{b} / \partial x^{\alpha}$. We are assuming that the variables $\theta^{a}$ are cyclic for the controlled Lagrangian. Correspondingly, we are assuming that $\tau_{\beta}^{a}$ depends only on $x^{\alpha}$ in this calculation and those that follow.

Using (2.4), we have

$$
\frac{\partial L}{\partial \dot{x}^{\alpha}}=g_{\alpha \beta} \dot{x}^{\beta}+g_{\alpha a} \dot{\theta}^{a}
$$

and

$$
\begin{aligned}
\frac{\partial L}{\partial x^{\alpha}}= & \frac{1}{2} g_{\beta \delta, \alpha} \dot{x}^{\beta} \dot{x}^{\delta}+g_{\delta b, \alpha} \dot{x}^{\delta} \dot{\theta}^{b} \\
& +\frac{1}{2} g_{a b, \alpha} \dot{\theta}^{a} \dot{\theta}^{b}-V_{, \alpha}
\end{aligned}
$$

where we again use commas to denote partial differentiation of the components of the metric tensor (mass matrix) and $V$; again, these are functions only of $x^{\alpha}$-and not of $\theta^{a}$ since the $\theta^{a}$ variables are assumed cyclic.

Using (2.16) in the first line and (2.17) in the second line of (2.15), the Euler-Lagrange expression $\mathcal{E}_{x}\left(L_{\tau, \sigma}\right)$ simplifies as follows:

$$
\begin{aligned}
\mathcal{E}_{x}\left(L_{\tau, \sigma}\right)= & \frac{d}{d t}\left[g_{\alpha a} \tau_{\beta}^{a} \dot{x}^{\beta}\right]-\left[g_{\delta b, \alpha} \dot{x}^{\delta} \tau_{\beta}^{b} \dot{x}^{\beta}\right. \\
& \left.+\frac{1}{2} g_{a b, \alpha}\left(2 \tau_{\beta}^{a} \dot{x}^{\beta} \dot{\theta}^{b}+\tau_{\beta}^{a} \dot{x}^{\beta} \tau_{\delta}^{b} \dot{x}^{\delta}\right)\right] \\
& -\frac{\partial L}{\partial \dot{\theta}^{b}}\left(x^{\delta}, \dot{x}^{\delta}, \dot{\theta}^{a}+\tau_{\beta}^{a} \dot{x}^{\beta}\right) \tau_{\delta, \alpha}^{b} \dot{x}^{\delta} \\
& +\frac{d}{d t}\left[\frac{\partial L}{\partial \dot{\theta}^{b}}\left(x^{\alpha}, \dot{x}^{\alpha}, \dot{\theta}^{a}+\tau_{\alpha}^{a} \dot{x}^{\alpha}\right) \tau_{\alpha}^{b}+\sigma_{a b} \tau_{\beta}^{a} \tau_{\alpha}^{b} \dot{x}^{\beta}\right] \\
& -\frac{\partial}{\partial x^{\alpha}}\left[\frac{1}{2} \sigma_{a b} \tau_{\delta}^{a} \tau_{\beta}^{b} \dot{x}^{\delta} \dot{x}^{\beta}\right] .
\end{aligned}
$$

Using the controlled conservation law (2.12) in the third and fourth lines, this becomes

$$
\begin{aligned}
\mathcal{E}_{x}\left(L_{\tau, \sigma}\right)= & \frac{d}{d t}\left[g_{\alpha a} \tau_{\beta}^{a} \dot{x}^{\beta}\right]-\left[g_{\delta b, \alpha} \dot{x}^{\delta} \tau_{\beta}^{b} \dot{x}^{\beta}\right. \\
& \left.+\frac{1}{2} g_{a b, \alpha}\left(2 \tau_{\beta}^{a} \dot{x}^{\beta} \dot{\theta}^{b}+\tau_{\beta}^{a} \dot{x}^{\beta} \tau_{\delta}^{b} \dot{x}^{\delta}\right)\right] \\
& -\tilde{J}_{b} \tau_{\delta, \alpha}^{b} \dot{x}^{\delta}+\frac{d}{d t}\left[\tilde{J}_{b} \tau_{\alpha}^{b}+\sigma_{a b} \tau_{\beta}^{a} \tau_{\alpha}^{b} \dot{x}^{\beta}\right] \\
& -\frac{\partial}{\partial x^{\alpha}}\left[\frac{1}{2} \sigma_{a b} \tau_{\delta}^{a} \tau_{\beta}^{b} \dot{x}^{\delta} \dot{x}^{\beta}\right] .
\end{aligned}
$$

Since $\theta$ is cyclic for the controlled Lagrangian, the controlled conserved quantity is actually conserved. Thus, the above expression becomes

$$
\begin{aligned}
\mathcal{E}_{x}\left(L_{\tau, \sigma}\right)= & \frac{d}{d t}\left[g_{\alpha a} \tau_{\beta}^{a} \dot{x}^{\beta}\right]-\left[g_{\delta b, \alpha} \dot{x}^{\delta} \tau_{\beta}^{b} \dot{x}^{\beta}\right. \\
& \left.+\frac{1}{2} g_{a b, \alpha}\left(2 \tau_{\beta}^{a} \dot{x}^{\beta} \dot{\theta}^{b}+\tau_{\beta}^{a} \dot{x}^{\beta} \tau_{\delta}^{b} \dot{x}^{\delta}\right)\right] \\
& +\tilde{J}_{b}\left[\tau_{\alpha, \delta}^{b}-\tau_{\delta, \alpha}^{b}\right] \dot{x}^{\delta}+\frac{d}{d t}\left[\sigma_{a b} \tau_{\beta}^{a} \tau_{\alpha}^{b} \dot{x}^{\beta}\right] \\
& -\frac{\partial}{\partial x^{\alpha}}\left[\frac{1}{2} \sigma_{a b} \tau_{\delta}^{a} \tau_{\beta}^{b} \dot{x}^{\delta} \dot{x}^{\beta}\right]
\end{aligned}
$$

Some Assumptions: Now we are ready to introduce some crucial assumptions that are designed to make the preceding Euler-Lagrange expression vanish. The first of these is the following.

Assumption $M-1: \tau_{\alpha}^{b}=-\sigma^{a b} g_{\alpha a}$.

This condition says, roughly speaking, that $\tau_{\alpha}^{b}$ are chosen to be the components of the "mechanical connection" formed out of $g$ and $g_{\sigma}$. Of course, the condition can be equivalently written as

$$
\sigma_{a b} \tau_{\alpha}^{b}=-g_{\alpha a}
$$

With Assumption M-1, the above expression becomes

$$
\begin{aligned}
\mathcal{E}_{x}\left(L_{\tau, \sigma}\right) & {\left[g_{\delta b, \alpha} \dot{x}^{\delta} \tau_{\beta}^{b} \dot{x}^{\beta}+\frac{1}{2} g_{a b, \alpha}\left(2 \tau_{\beta}^{a} \dot{x}^{\beta} \dot{\theta}^{b}+\tau_{\beta}^{a} \dot{x}^{\beta} \tau_{\delta}^{b} \dot{x}^{\delta}\right)\right] } \\
= & +\tilde{J}_{b}\left[\tau_{\alpha, \delta}^{b}-\tau_{\delta, \alpha}^{b}\right] \dot{x}^{\delta}-\frac{\partial}{\partial x^{\alpha}}\left[\frac{1}{2} \sigma_{\alpha b} \tau_{\delta}^{a} \tau_{\beta}^{b} \dot{x}^{\delta} \dot{x}^{\beta}\right] \\
= & {\left[g_{\delta b, \alpha} \dot{x}^{\delta} \sigma^{b c} g_{\beta c} \dot{x}^{\beta}-\frac{1}{2} g_{a b, \alpha}\left(2 \tau_{\beta}^{a} \dot{x}^{\beta} \dot{\theta}^{b}+\tau_{\beta}^{a} \dot{x}^{\beta} \tau_{\delta}^{b} \dot{x}^{\delta}\right)\right] } \\
& +\tilde{J}_{b}\left[\tau_{\alpha, \delta}^{b}-\tau_{\delta, \alpha}^{b}\right] \dot{x}^{\delta}-\frac{\partial}{\partial x^{\alpha}}\left[\frac{1}{2} g_{\delta b} \sigma^{b c} g_{\beta c} \dot{x}^{\delta} \dot{x}^{\beta}\right] \\
= & -\frac{1}{2}\left[g_{\delta b} \sigma_{, \alpha}^{b c} g_{\beta c} \dot{x}^{\delta} \dot{x}^{\beta}+g_{a d, \alpha}\left(2 \tau_{\beta}^{a} \dot{x}^{\beta} \dot{\theta}^{d}+\tau_{\beta}^{a} \dot{x}^{\beta} \tau_{\delta}^{d} \dot{x}^{\delta}\right)\right] \\
& +\tilde{J}_{b}\left[\tau_{\alpha, \delta}^{b}-\tau_{\delta, \alpha}^{b}\right] \dot{x}^{\delta} .
\end{aligned}
$$

Using the controlled conserved quantity we get

$$
\dot{\theta}^{d}=g^{d b}\left(\tilde{J}_{b}-\left[g_{\delta b}+g_{b e} \tau_{\delta}^{e}\right] \dot{x}^{\delta}\right)
$$


and hence the preceding expression for $\mathcal{E}_{x}\left(L_{\tau, \sigma}\right)$ becomes

$$
\begin{aligned}
\mathcal{E}_{x}\left(L_{\tau, \sigma}\right) & \\
= & -\frac{1}{2}\left[g_{\delta b} \sigma_{, \alpha}^{b c} g_{\beta c}-2 g_{a d, \alpha} \tau_{\beta}^{a} g^{b d} g_{\delta b}-g_{a d, \alpha} \tau_{\beta}^{a} \tau_{\delta}^{d}\right] \dot{x}^{\delta} \dot{x}^{\beta} \\
& +\tilde{J}_{b}\left[\tau_{\alpha, \delta}^{b}-\tau_{\delta, \alpha}^{b}-g^{d b} g_{a d, \alpha} \tau_{\delta}^{a}\right] \dot{x}^{\delta}
\end{aligned}
$$

Using the Assumption M-1 again to eliminate $\tau$ in the first line, we get

$$
\begin{aligned}
\mathcal{E}_{x}( & \left.L_{\tau, \sigma}\right) \\
= & -\frac{1}{2} g_{\delta b} g_{\beta c}\left[\sigma_{, \alpha}^{b c}+2 g_{a d, \alpha} \sigma^{a c} g^{b d}-g_{a d, \alpha} \sigma^{a c} \sigma^{d b}\right] \dot{x}^{\delta} \dot{x}^{\beta} \\
& +\tilde{J}_{b}\left[\tau_{\alpha, \delta}^{b}-\tau_{\delta, \alpha}^{b}-g^{d b} g_{a d, \alpha} \tau_{\delta}^{a}\right] \dot{x}^{\delta} \\
= & \left.\frac{1}{2} g_{\delta b} g_{\beta c} \sigma^{a c}\left[\sigma^{b d}\left(\sigma_{a d, \alpha}+g_{a d, \alpha}\right)-2 g^{b d} g_{a d, \alpha}\right)\right] \dot{x}^{\delta} \dot{x}^{\beta} \\
& +\tilde{J}_{b}\left[\tau_{\alpha, \delta}^{b}-\tau_{\delta, \alpha}^{b}-g^{d b} g_{a d, \alpha} \tau_{\delta}^{a}\right] \dot{x}^{\delta}
\end{aligned}
$$

Now we are ready to state our second two assumptions.

Assumption M-2: $\sigma^{b d}\left(\sigma_{a d, \alpha}+g_{a d, \alpha}\right)=2 g^{b d} g_{a d, \alpha}$.

Assumption M-3: $\tau_{\alpha, \delta}^{b}-\tau_{\delta, \alpha}^{b}-g^{d b} g_{a d, \alpha} \tau_{\delta}^{a}=0$.

The following theorem gives sufficient conditions for matching.

Theorem 2.2 (First Matching Theorem): Under Assumptions M-1-M-3, the Euler-Lagrange equations for the controlled Lagrangian $L_{\tau, \sigma}$ given by (2.3) coincide with the controlled Euler-Lagrange equations (2.6).

Simplified Matching Assumptions: Consider the following:

1) $\sigma_{a b}=\sigma g_{a b}$ for a constant $\sigma$ (this defines $\sigma_{a b}$ );

2) $g_{a b}$ is independent of $x^{\alpha}$ (a condition on the metric tensor);

3) $\tau_{\alpha}^{b}=-(1 / \sigma) g^{a b} g_{\alpha a}$ (this defines $\left.\tau_{\alpha}^{b}\right)$;

4) $g_{\alpha a, \delta}=g_{\delta a, \alpha}$ (a second condition on the metric).

If these hold, then all three of M-1-M-3 hold, so we have matching. The second and fourth of the Simplified Matching Assumptions imply that the mechanical connection $g^{a b} g_{a \alpha}$ for the given system is flat, i.e., systems that satisfy the Simplified Matching Assumptions lack gyroscopic forces. The $\sigma$ in this case is a free variable and can be interpreted as the control gain. These simplified conditions hold for the case of the inverted pendulum on a cart discussed in Section I-B.

As we have mentioned, this theorem is generalized to incorporate the $g_{\rho}$ terms in companion papers, so that we will find a more general matching theorem.

The following remark illustrates that care must be taken in relating the controlled to the uncontrolled case: if one sets $\sigma$ equal to the identity in the first simplified matching assumption, the $\tau$-horizontal and $\tau$-vertical projections (2.9) do not reduce to the uncontrolled projections (2.7), but to the trivial projections. Rather, to recover the original projections, the one form $\tau$ should be taken to be trivial.

\section{The Control LAW AND StABILIZATION}

Now that we have achieved matching in the Euler-Lagrange equations for the shape variables, we can proceed to determine the control law and then conditions under which stabilization is achieved. We continue to restrict to the case in which $g_{\rho}=g$.

\section{A. Determination of the Control Law}

The control law is determined from the difference between the $\theta^{a}$ Euler-Lagrange equations for the controlled and the uncontrolled Lagrangians. In our case we have arranged to not break the symmetry, and so we may determine the control law from the difference between the two conservation laws.

To do this, we start with the relation

$$
\tilde{J}_{a}=J_{a}+g_{a b} \tau_{\alpha}^{b} \dot{x}^{\alpha}
$$

and since $\tilde{J}_{a}$ is conserved, we may write

$$
\begin{aligned}
u_{a} & =\frac{d}{d t} J_{a}=\frac{d}{d t} \tilde{J}_{a}-\frac{d}{d t}\left(g_{a b} \tau_{\alpha}^{b} \dot{x}^{\alpha}\right)=-\frac{d}{d t}\left(g_{a b} \tau_{\alpha}^{b} \dot{x}^{\alpha}\right) \\
& =-\left(g_{a b, \delta} \tau_{\alpha}^{b} \dot{x}^{\alpha} \dot{x}^{\delta}+g_{a b} \tau_{\alpha, \delta}^{b} \dot{x}^{\alpha} \dot{x}^{\delta}+g_{a b} \tau_{\alpha}^{b} \ddot{x}^{\alpha}\right) .
\end{aligned}
$$

Our final control law does not depend on accelerations: we eliminate the accelerations $\ddot{x}^{\alpha}$ from this expression for the control by making use of the fact that the Euler-Lagrange equations for $x$ hold (for both $L$ and $L_{\tau, \sigma}$ ). Using (2.4), and the fact that $\theta^{a}$ is cyclic, the explicit $x^{\alpha}$-Euler-Lagrange equation is

$$
\begin{gathered}
g_{\alpha \beta} \ddot{x}^{\beta}+\left(g_{\alpha \beta, \gamma}-\frac{1}{2} g_{\beta \gamma, \alpha}\right) \dot{x}^{\beta} \dot{x}^{\gamma}+\left(g_{\alpha a, \gamma}-g_{\gamma a, \alpha}\right) \dot{x}^{\gamma} \dot{\theta}^{a} \\
-\frac{1}{2} g_{a b, \alpha} \dot{\theta}^{a} \dot{\theta}^{b}+g_{\alpha a} \ddot{\theta}^{a}=-\frac{\partial V}{\partial x^{\alpha}} .
\end{gathered}
$$

Next, we use the $\theta^{a}$-Euler-Lagrange equation for the controlled Lagrangian to determine $\ddot{\theta}^{a}$. That is, we simply write out the conservation law for $\tilde{J}_{a}$. Setting the time derivative of $\tilde{J}_{a}$ from (2.12) equal to zero, we get

$$
\begin{gathered}
\left(g_{\alpha a, \delta}+g_{a b, \delta} \tau_{\alpha}^{b}+g_{a b} \tau_{\alpha, \delta}^{b}\right) \dot{x}^{\alpha} \dot{x}^{\delta}+g_{\alpha a} \ddot{x}^{\alpha} \\
+g_{a b, \delta} \dot{\theta}^{a} \dot{x}^{\delta}+g_{a b} \ddot{\theta}^{b}+g_{a b} \tau_{\alpha}^{b} \ddot{x}^{\alpha}=0
\end{gathered}
$$

and hence

$$
\begin{aligned}
\ddot{\theta}^{d}=-g^{d a}[ & \left(g_{\alpha a, \delta}+g_{a b, \delta} \tau_{\alpha}^{b}+g_{a b} \tau_{\alpha, \delta}^{b}\right) \dot{x}^{\alpha} \dot{x}^{\delta} \\
& \left.+g_{\alpha a} \ddot{x}^{\alpha}+g_{a b, \delta} \dot{\theta}^{b} \dot{x}^{\delta}\right]-\tau_{\alpha}^{d} \ddot{x}^{\alpha} .
\end{aligned}
$$

Substituting (3.5) into (3.3) gives

$$
\begin{aligned}
& \left(g_{\alpha \beta}-g_{\alpha d} \tau_{\beta}^{d}-g_{\alpha d} g^{d a} g_{\beta a}\right) \ddot{x}^{\beta} \\
& +\left[g_{\alpha \beta, \gamma}-\frac{1}{2} g_{\beta \gamma, \alpha}-g_{\alpha d} g^{d a}\right. \\
& \left.\quad \times\left(g_{\beta a, \gamma}+g_{a b, \gamma} \tau_{\beta}^{b}+g_{a b} \tau_{\beta, \gamma}^{b}\right)\right] \dot{x}^{\beta} \dot{x}^{\gamma} \\
& +\left(g_{\alpha a, \gamma}-g_{\gamma a, \alpha}-g_{\alpha d} g^{d b} g_{a b, \gamma}\right) \dot{x}^{\gamma} \dot{\theta}^{a} \\
& -\frac{1}{2} g_{a b, \alpha} \dot{\theta}^{a} \dot{\theta}^{b}=-\frac{\partial V}{\partial x^{\alpha}} .
\end{aligned}
$$

The control law is now determined by substituting this equation into (3.2). So far, our derivation is rather general, but we can simplify things somewhat by using our assumptions. Using M-1 and M-3, (3.6) simplifies to

$$
\begin{aligned}
& \left(g_{\alpha \beta}+g_{\alpha d}\left[\sigma^{d a}-g^{d a}\right] g_{\beta a}\right) \ddot{x}^{\beta} \\
& \quad+\left[g_{\alpha \beta, \gamma}-\frac{1}{2} g_{\beta \gamma, \alpha}-g_{\alpha d} g^{d a} g_{\beta a, \gamma}-g_{\alpha d} \tau_{\beta, \gamma}^{d}\right] \dot{x}^{\beta} \dot{x}^{\gamma} \\
& \quad+\left(g_{\alpha a, \gamma}-g_{\gamma a, \alpha}-g_{\alpha d} g^{d b} g_{a b, \gamma}\right) \dot{x}^{\gamma} \dot{\theta}^{a} \\
& \quad-\frac{1}{2} g_{a b, \alpha} \dot{\theta}^{a} \dot{\theta}^{b}=-\frac{\partial V}{\partial x^{\alpha}}
\end{aligned}
$$


Now let

$$
A_{\alpha \beta}=g_{\alpha \beta}+g_{\alpha d}\left[\sigma^{d a}-g^{d a}\right] g_{\beta a} .
$$

Assume that this matrix is invertible and let $A^{\alpha \beta}$ denote its inverse. Hence

$$
\begin{aligned}
\ddot{x}^{\delta}= & -A^{\delta \alpha}\left[g_{\alpha \beta, \gamma}-\frac{1}{2} g_{\beta \gamma, \alpha}-g_{\alpha d} g^{d a} g_{\beta a, \gamma}-g_{\alpha d} \tau_{\beta, \gamma}^{d}\right] \dot{x}^{\beta} \dot{x}^{\gamma} \\
& -A^{\delta \alpha}\left(g_{\alpha a, \gamma}-g_{\gamma a, \alpha}-g_{\alpha d} g^{d b} g_{\alpha b, \gamma}\right) \dot{x}^{\gamma} \dot{\theta}^{a} \\
& +A^{\delta \alpha} \frac{1}{2} g_{a b, \alpha} \dot{\theta}^{a} \dot{\theta}^{b}-A^{\delta \alpha} \frac{\partial V}{\partial x^{\alpha}}
\end{aligned}
$$

Notice also that under M-3 $g_{a b, \delta} \tau_{\alpha}^{b}$ is skew-symmetric in the $\delta, \alpha$ indexes and hence the first term on the right-hand side of the control law (3.2) vanishes. Substitution of (3.9) into the control law gives

$$
\begin{aligned}
u_{a}=- & \left\{g_{a b} \tau_{\beta, \gamma}^{b}-g_{a b} \tau_{\delta}^{b} A^{\delta \alpha}\left[g_{\alpha \beta, \gamma}-\frac{1}{2} g_{\beta \gamma, \alpha}\right.\right. \\
& \left.\left.-g_{\alpha d} g^{d a} g_{\beta a, \gamma}-g_{\alpha d} \tau_{\beta, \gamma}^{d}\right]\right\} \dot{x}^{\beta} \dot{x}^{\gamma} \\
& -g_{a b} \tau_{\delta}^{b} A^{\delta \alpha}\left[-\left(g_{\alpha c, \gamma}-g_{\gamma c, \alpha}-g_{\alpha d} g^{d b} g_{c b, \gamma}\right) \dot{x}^{\gamma} \dot{\theta}^{c}\right. \\
& \left.+\frac{1}{2} g_{c b, \alpha} \dot{\theta}^{c} \dot{\theta}^{b}-\frac{\partial V}{\partial x^{\alpha}}\right]
\end{aligned}
$$

One may eliminate $\dot{\theta}^{a}$ if desired by making use of the relation

$$
\begin{aligned}
\dot{\theta}^{b} & =g^{a b} \tilde{J}_{a}-\left(g^{a b} g_{\alpha a}+\tau_{\alpha}^{b}\right) \dot{x}^{\alpha} \\
& =g^{a b} \tilde{J}_{a}-\left[g^{a b}-\sigma^{a b}\right] g_{\alpha a} \dot{x}^{\alpha} .
\end{aligned}
$$

Under the Simplified Matching Assumptions given after the First Matching Theorem 2.2, the coefficients of the terms multiplying $\dot{\theta}$ vanish and the formula for the control becomes

$$
\begin{aligned}
u_{a}= & \frac{1}{\sigma}\left\{g_{\beta a, \gamma}-g_{\delta a} A^{\delta \alpha}\left[g_{\alpha \beta, \gamma}-\frac{1}{2} g_{\beta \gamma, \alpha}\right.\right. \\
& \left.\left.-\left(1-\frac{1}{\sigma}\right) g_{\alpha d} g^{d a} g_{\beta a, \gamma}\right]\right\} \dot{x}^{\beta} \dot{x}^{\gamma} \\
& -\frac{1}{\sigma} g_{\delta a} A^{\delta \alpha} \frac{\partial V}{\partial x^{\alpha}}
\end{aligned}
$$

where

$$
A_{\alpha \beta}=g_{\alpha \beta}-g_{\alpha d}\left(1-\frac{1}{\sigma}\right) g^{d a} g_{\beta a} .
$$

Note that the control law only involves position and velocity feedback, not acceleration feedback.

Proposition 3.1: Suppose the conditions of Theorem 2.2 hold [i.e., the First Matching Theorem holds with the controlled Lagrangian $L_{\tau, \sigma}$ defined by (2.3)]. Suppose that $A_{\alpha \beta}$ defined by (3.8) is invertible. Then, (3.10) provides the corresponding feedback control law $u$ as a function of positions and velocities only (i.e., there is no acceleration feedback). Furthermore, in the case that the Simplified Matching Assumptions hold, this feedback law simplifies to that given in (3.12) which is independent of the velocities of the symmetry variables.

The above calculations make intrinsic geometric sense. For example, and we shall need this remark, the matrix $A_{\alpha \beta}$ may be interpreted as the components of the horizontal metric (the shape space projected metric) for the controlled Lagrangian. Under Assumption M-1, the formula for the horizontal part of the metric is given by [see (2.9)]

$$
\operatorname{Hor}_{\tau}\left(v_{q}\right)=\left(\dot{x}^{\alpha},\left(\sigma^{a b}-g^{a b}\right) g_{\alpha b} \dot{x}^{\alpha}\right) .
$$

Intrinsically, the calculation of the horizontal kinetic energy expression is as follows (see the proof of the first matching theorem):

$$
\begin{aligned}
K_{\text {hor }_{\tau}}\left(v_{q}\right) & :=\frac{1}{2}\left[g_{\sigma}\left(\operatorname{Hor}_{\tau} v_{q}, \operatorname{Hor}_{\tau} v_{q}\right)\right] \\
& =\frac{1}{2}\left[g_{\sigma}\left(\operatorname{Hor}(v)-\tau(v)_{Q}, \operatorname{Hor}(v)-\tau(v)_{Q}\right)\right] \\
& =\frac{1}{2}\left[g(\operatorname{Hor}(v), \operatorname{Hor}(v))+g_{\sigma}\left(\tau(v)_{Q}, \tau(v)_{Q}\right)\right] .
\end{aligned}
$$

In coordinates

$$
\begin{aligned}
& \frac{1}{2} g(\operatorname{Hor}(v), \operatorname{Hor}(v)) \\
& \quad=\frac{1}{2} g_{\alpha \beta} \dot{x}^{\alpha} \dot{x}^{\beta}-g_{a \alpha} g^{a b} g_{b \beta} \dot{x}^{\alpha} \dot{x}^{\beta}+\frac{1}{2} g_{a \alpha} g^{a b} g_{b \beta} \dot{x}^{\alpha} \dot{x}^{\beta} \\
& \quad=\frac{1}{2}\left(g_{\alpha \beta}-g_{a \alpha} g^{a b} g_{b \beta}\right) \dot{x}^{\alpha} \dot{x}^{\beta}
\end{aligned}
$$

while under Assumption M-1 we have

$$
\begin{aligned}
\frac{1}{2} g_{\sigma}\left(\tau(v)_{Q}, \tau(v)_{Q}\right) & =\frac{1}{2} \sigma_{a b}\left(-\sigma^{a d} g_{d \alpha}\right)\left(-\sigma^{b e} g_{e, \beta}\right) \dot{x}^{\alpha} \dot{x}^{\beta} \\
& =\frac{1}{2} \sigma^{a b} g_{a \alpha} g_{b \beta} \dot{x}^{\alpha} \dot{x}^{\beta}
\end{aligned}
$$

Adding these gives the following.

Proposition 3.2: The $\tau$-horizontal kinetic energy is given by

$$
K_{\text {hor }_{\tau}}\left(v_{q}\right)=\frac{1}{2} A_{\alpha \beta} \dot{x}^{\alpha} \dot{x}^{\beta} \text {. }
$$

\section{B. Stabilization of Relative Equilibria}

Recall that a relative equilibrium for a mechanical system with symmetry is a solution of the equations that is simultaneously a one-parameter group orbit. When the symmetry groups are Euclidean groups, examples of these are uniformly rotating and translating solutions. A general introduction to and basic facts about relative equilibria can be found in [31].

Since $\tau$ is horizontal, for any Lie algebra element $\xi \in \mathfrak{g}$, we have $\tau\left(\xi_{Q}(q)\right)=0$. This implies the identity $L\left(\xi_{Q}(q)\right)=$ $L_{\tau, \sigma}\left(\xi_{Q}(q)\right)$.

Given a Lagrangian $L$ and a Lie algebra element $\xi \in \mathfrak{g}$, the function $L_{\xi}(q):=L\left(\xi_{Q}(q)\right)$ is called the associated locked Lagrangian. Thus, from the identity $L\left(\xi_{Q}(q)\right)=L_{\tau, \sigma}\left(\xi_{Q}(q)\right)$ noted in the preceding paragraph, we conclude that $L$ and $L_{\tau, \sigma}$ have the same locked Lagrangian.

It is known that relative equilibria are the critical points of the locked Lagrangian (see [27, Prop. 2.3] and [39]); this is a generalization of the classical criterion, going back to Routh around 1850, which states that relative equilibrium are critical points of either the amended or augmented potential. ${ }^{1}$ Intuitively, the modification of the Lagrangian to the controlled Lagrangian, while affecting the kinetic energy, does not affect the augmented potential. Therefore, we conclude the following.

Proposition 3.3: The relative equilibria for $L$ and $L_{\tau, \sigma}$ are the same.

\footnotetext{
${ }^{1}$ The amended potential is recalled in (3.19). The augmented potential is given by a similar formula using generalized angular velocities rather than angular momenta.
} 
One can now use the energy momentum method [36], [31], especially its Lagrangian formulation [27], [39] to ascertain stability.

From general considerations, we know that a relative equilibrium is a fixed point of the reduced dynamics on shape space. As such, it must satisfy $\dot{x}^{\alpha}=0$. To emphasize that this corresponds to an equilibrium value, we shall sometimes write the equilibrium point as $x_{e}$, or in coordinates, $x_{e}^{\alpha}$. Consequently, from (2.13), the momentum at a relative equilibrium is the same for the free and for the controlled system. We call this value $\mu$, with components $\mu_{a}$, or if there is danger of confusion, by $\mu_{a}^{e}$, where the superscript $e$ refers to the equilibrium value.

Next, we give a criterion for stability of control systems that are described by a controlled Lagrangian of the form $L_{\tau, \sigma}$ given by (2.3).

Theorem 3.4: Suppose the conditions of Theorem 2.2 hold (i.e., the First Matching Theorem holds with the controlled Lagrangian $L_{\tau, \sigma}$ ). A point $x_{e}^{\alpha}$ is a relative equilibrium if and only if it is a critical point of $V_{\mu}$ where $\mu$ is the value of the equilibrium momentum and where $V_{\mu}$ is the amended potential defined by

$$
V_{\mu}\left(x^{\alpha}\right)=V\left(x^{\alpha}\right)+\frac{1}{2} g^{a b} \mu_{a} \mu_{b}
$$

Then, the system is stabilized about the given equilibrium if the second variation of

$$
E_{\mu}:=\frac{1}{2} A_{\alpha \beta} \dot{x}^{\alpha} \dot{x}^{\beta}+V_{\mu}
$$

[as a function of the variables $x^{\alpha}$ and where $A_{\alpha \beta}$ is defined in (3.8)] evaluated at the equilibrium is definite.

Proof: The proof proceeds in a standard way following the energy-momentum method by showing that $E_{\mu}$ is the reduced expression for the energy of the system. This calculation is done for a general Lagrangian in, for example, [31], and is here applied to $L_{\tau, \sigma}$ using the fact, proved earlier [see (3.18)] that the horizontal part of the $\tau$-kinetic energy is, under Assumption M-1, (1/2) $A_{\alpha \beta} \dot{x}^{\alpha} \dot{x}^{\beta}$ to get the result.

Remarks:

1) In the special case when $g_{a b}$ is constant, the extra term in the amended potential is a constant and so does not contribute to the second variation.

2) One has stability modulo the (Abelian) group $G$ in the unreduced space. (See [29] for more sophisticated applications in which one gets stability modulo a subgroup.) Note further that since the equilibrium of interest for a balance system is a maximum of the potential energy and we are not modifying the potential here, our controller will in general lead to a local maximum in the reduced space. As discussed in Section $\mathrm{V}$ the addition of active dissipation then leads to a robust asymptotically stable equilibrium.

3) Note that the energy momentum function $E_{\mu}$ depends on the system gains.

4) If the system has an additional symmetry group, then one can, of course, use the energy momentum method to study stability of relative equilibria for that group. We shall see an example of a system with another symmetry group in the inverted spherical pendulum below. The controlled Euler-Poincaré equations may also be viewed this way-these are studied in companion papers.

\section{INVERTED PENDULA}

In this section we illustrate the results of the preceding sections with two examples. In the first subsection, we re-examine the inverted pendulum on a cart and show that a direct application of the matching theorem of Section II and stabilization theorem of Section III produce the stabilizing control law derived in Section I-B. In the second subsection we show how to apply these techniques to the case of the inverted spherical pendulum.

\section{A. Reprise of the Inverted Pendulum on a Cart}

Recall that the configuration space is $Q=S \times G$ where $S=$ $S^{1}$ describes the angle $\phi$ of the pendulum and $G=\mathbb{R}$ describes the position $s$ of the cart. When using the general theory, keep in mind that $x$ in the general theory corresponds to $\phi$ here and that $\theta$ in the general theory corresponds to $s$ here.

The Lagrangian: The Lagrangian is

$$
L(\phi, \dot{\phi}, \dot{s})=\frac{1}{2}\left[\begin{array}{ll}
\dot{\phi} & \dot{s}
\end{array}\right]\left[\begin{array}{cc}
\alpha & \beta \cos \phi \\
\beta \cos \phi & \gamma
\end{array}\right]\left[\begin{array}{c}
\dot{\phi} \\
\dot{s}
\end{array}\right]+D \cos \phi
$$

where $\alpha, \beta, \gamma$, and $D$ are as defined in Section I-B.

Controlled Lagrangian and Matching: We apply Theorem 2.2 to get the controlled Lagrangian that matches the controlled Euler-Lagrange equations (1.10). Since $G=\mathbb{R}$ is one-dimensional, both $g_{a b}$ and $\sigma_{a b}$ are scalars. We have that $g_{a b}=\gamma$. Let $\sigma_{a b}=\sigma \gamma$ where $\sigma$ is a dimensionless scalar. Since $g_{a b}$ is a constant, to satisfy M-2 we should also take $\sigma$ to be a constant. To satisfy M-1, we choose

$$
\tau_{\phi}^{s}=-\frac{1}{\sigma \gamma} \beta \cos \phi
$$

M-3 is then trivially satisfied, and the controlled Lagrangian provides matching. In fact, the Simplified Matching Assumptions hold. Following (2.2) the controlled Lagrangian is

$$
\begin{aligned}
L_{\tau, \sigma}= & L\left(\phi, \dot{\phi}, \dot{s}-\frac{\beta}{\sigma \gamma} \cos \phi \dot{\phi}\right)+\frac{1}{2} \sigma \gamma\left(\frac{\beta}{\sigma \gamma} \cos \phi \dot{\phi}\right)^{2} \\
& +D \cos \phi \\
= & \frac{1}{2}\left(\alpha \dot{\phi}^{2}+2 \beta \cos \phi\left(\dot{s}-\frac{\beta}{\sigma \gamma} \cos \phi \dot{\phi}\right) \dot{\phi}\right. \\
& \left.\quad+\gamma\left(\dot{s}-\frac{\beta}{\sigma \gamma} \cos \phi \dot{\phi}\right)^{2}\right)+\frac{1}{2} \frac{\beta^{2}}{\sigma \gamma} \cos ^{2} \phi \dot{\phi}^{2} \\
& +D \cos \phi .
\end{aligned}
$$

Note that defining $\kappa:=-1 / \sigma$ and substituting for $\sigma$ in (4.1), we recover the controlled Lagrangian of (1.11), where $k(\phi)$ is defined by (1.17).

Control Law: Using (3.2), the control law is

$$
u=\frac{d}{d t}\left(\frac{\beta}{\sigma} \cos \phi \dot{\phi}\right)=-\frac{d}{d t}(\kappa \beta \cos \phi \dot{\phi}) .
$$

We can use the general formula (3.12) to calculate this control law with the acceleration term $\ddot{\phi}$ eliminated. 


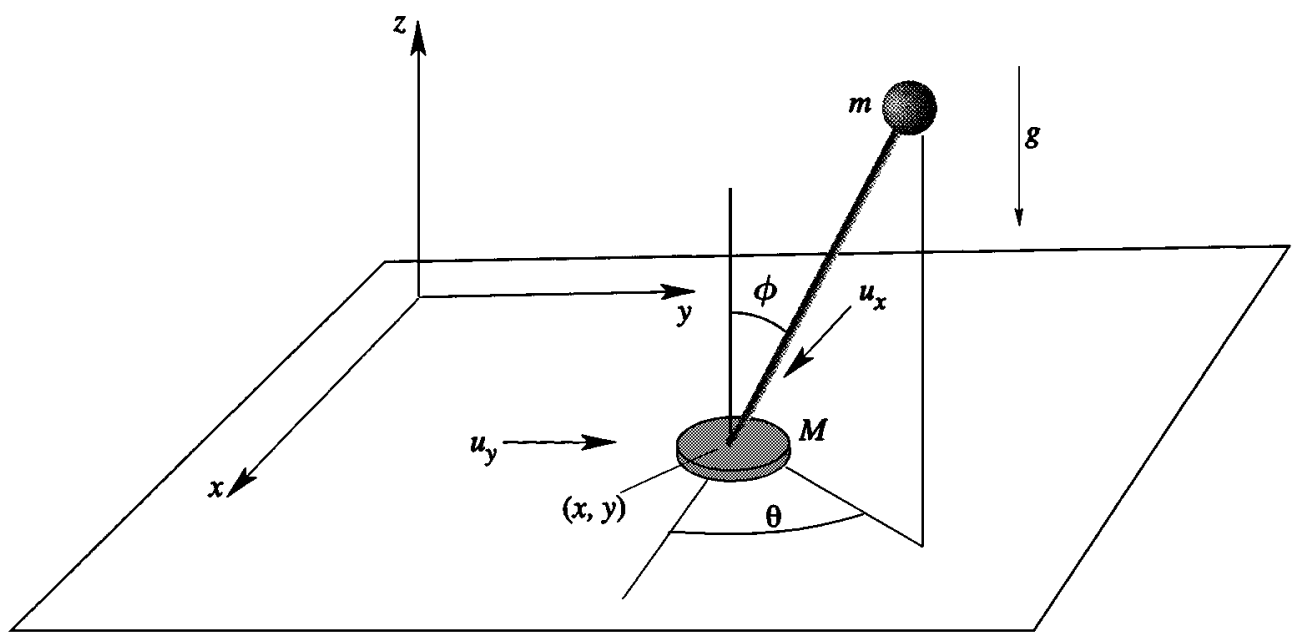

Fig. 2. The inverted spherical pendulum on a cart.

In this case, $-g_{a b} \tau_{\mu, \delta}^{b} \dot{x}^{\mu} \dot{x}^{\delta}=\kappa \beta \sin \phi \dot{\phi}^{2}$, the matrix (3.8) becomes the scalar

$$
A=\alpha-\frac{\beta^{2}}{\gamma}(1+\kappa) \cos ^{2} \phi
$$

and

$$
\begin{aligned}
g_{a b} \tau_{\delta}^{b}\left[\left(g_{\mu \nu, \gamma}-\frac{1}{2} g_{\nu \gamma, \mu}-g_{\mu c} \tau_{\nu, \gamma}^{c}-g_{\mu c} g^{c b} g_{\nu b, \gamma}\right)\right. \\
\left.\times \dot{x}^{\nu} \dot{x}^{\gamma}+V_{, \mu}\right] \\
=(\kappa \beta \cos \phi)\left[\left(\frac{\beta^{2}}{\gamma} \cos \phi \sin \phi+\frac{\beta^{2} \kappa}{\gamma} \cos \phi \sin \phi\right)\right. \\
\left.\times \dot{\phi}^{2}+D \sin \phi\right] .
\end{aligned}
$$

Substituting into the general formula (3.12), we obtain the nonlinear pendulum-cart control law (1.18).

Stabilization: Following Theorem 3.4, the relative equilibrium $\phi=\dot{\phi}=\dot{s}=0$ is stable if the second variation of

$$
\frac{1}{2}\left(\alpha+\beta^{2} \cos ^{2} \phi\left(\frac{1}{\sigma \gamma}-\frac{1}{\gamma}\right)\right) \dot{\phi}^{2}-D \cos \phi
$$

is definite when evaluated at this equilibrium. This requires that the matrix

$$
\left(\begin{array}{cc}
D & 0 \\
0 & \alpha+\beta^{2}\left(\frac{1}{\sigma \gamma}-\frac{1}{\gamma}\right)
\end{array}\right)
$$

be positive or negative definite. Since $D=-m g l<0$, the matrix will be negative definite if

$$
\alpha+\beta^{2}\left(\frac{1}{\sigma \gamma}-\frac{1}{\gamma}\right)<0
$$

i.e., if

$$
\frac{1}{\sigma}<\frac{\beta^{2}-\alpha \gamma}{\beta^{2}}
$$

Equivalently, using $\kappa=-1 / \sigma$, the relative equilibrium is stable if

$$
\kappa>\frac{\alpha \gamma-\beta^{2}}{\beta^{2}}=\frac{M}{m}
$$

which is the stability condition (1.19).

\section{B. The Spherical Pendulum}

In this section we consider the controlled spherical pendulum on a cart in the $x y$-plane. This generalizes the planar pendulum example and provides a highly nontrivial example of matching and stabilization in the case where we only need a controlled Lagrangian of the form $L_{\tau, \sigma}$. In this case we have independent controls that can move the cart in the $x$ and $y$ directions.

Consider then a spherical pendulum with bob of mass $m$ on a movable base of mass $M$, as in Fig. 2. The base is idealized to be a point (or a symmetric planar body) as this simplifies the calculations without affecting the essential dynamics.

The Lagrangian: The free Lagrangian for the spherical pendulum on a cart is

$$
\begin{aligned}
L= & \frac{1}{2} M\left(\dot{x}^{2}+\dot{y}^{2}\right)+\frac{1}{2} m\left(\dot{x}^{2}+\dot{y}^{2}+r^{2} \dot{\phi}^{2}+r^{2} \sin ^{2} \phi \dot{\theta}^{2}\right. \\
& +2 r \cos \phi \dot{\phi}(\dot{x} \cos \theta+\dot{y} \sin \theta) 2 r \sin \phi \dot{\theta} \\
& \times(-\dot{x} \sin \theta+\dot{y} \cos \theta))+m g r(1-\cos \phi)
\end{aligned}
$$

where $\phi$ and $\theta$ are spherical coordinates measured in a frame with origin fixed on the (point) cart, but with orientation that remains fixed with respect to inertial space. $\phi$ represents the deflection from the vertical while $\theta$ represents the angle between the pendulum and the $x$-axis. The controlled equations are the Lagrangian equations with control forces $u_{x}$ and $u_{y}$ in the $x$ and $y$ equations, respectively. Note that the Lagrangian is cyclic in $x$ and $y$. However, the system is in fact $S E(2)$ invariant, as one would expect physically.

Consider the action of $S E(2)$ on $\mathbb{R}^{2} \times S^{1}$ given by

$$
\begin{aligned}
(x, y, \theta) \rightarrow & (x \cos \alpha-y \sin \alpha+a, \\
& x \sin \alpha+y \cos \alpha+b, \theta+\alpha)
\end{aligned}
$$


with induced action on $\operatorname{TSE}(2)$

$$
(\dot{x}, \dot{y}, \dot{\theta}) \rightarrow(\dot{x} \cos \alpha-\dot{y} \sin \alpha, \dot{x} \sin \alpha+\dot{y} \cos \alpha+b, \dot{\theta}) \text {. }
$$

A computation shows that the Lagrangian is indeed invariant under this action (thus giving rise to three conservation laws).

However, for the purposes of applying the theory discussed above we will assume the symmetry directions are the $x$ and $y$ directions ignoring for the moment the additional $S^{1}$ symmetry. We shall return to this later in this section. The key point here is that the controls act in the $x$ and $y$ directions and pick out the part of the symmetry group to be used in the matching theory. The remainder of the group is dealt with when doing stabilization.

Controlled Lagrangian and Matching: Note that twice the kinetic energy for the spherical pendulum on the cart can be written as shown in (4.5) at the bottom of the page. So, it can easily be seen that $g_{a b}$ is constant and, in addition, since

$$
\begin{aligned}
\frac{\partial}{\partial \theta}(\cos \phi \cos \theta) & =\frac{\partial}{\partial \phi}(-\sin \phi \sin \theta) \\
\frac{\partial}{\partial \theta}(\cos \phi \sin \theta) & =\frac{\partial}{\partial \phi}(\sin \phi \cos \theta)
\end{aligned}
$$

$g_{a \alpha, \delta}=g_{\delta a, \alpha}$ holds. We choose $\sigma_{a b}=\sigma g_{a b}$, where $\sigma$ is a constant, and $\tau_{\alpha}^{b}=-(1 / \sigma) g^{a b} g_{\alpha a}$. Then, Assumptions M-1-M-3 all hold (since the Simplified Matching Assumptions hold) and we get matching by Theorem 2.2. In this case, we have

$$
\begin{aligned}
\tau_{\phi}^{x} & =-\frac{m r}{\sigma(M+m)} \cos \phi \cos \theta \\
\tau_{\theta}^{x} & =-\frac{m r}{\sigma(M+m)}(-\sin \phi \sin \theta) \\
\tau_{\phi}^{y} & =-\frac{m r}{\sigma(M+m)} \cos \phi \sin \theta \\
\tau_{\theta}^{y} & =-\frac{m r}{\sigma(M+m)} \sin \phi \cos \theta .
\end{aligned}
$$

Using Theorem 2.1, the controlled Lagrangian is given by the free Lagrangian with velocity shifts

$$
\begin{aligned}
& \dot{x} \rightarrow \dot{x}-\frac{m r}{\sigma(M+m)}(\cos \phi \cos \theta \dot{\phi}-\sin \phi \sin \theta \dot{\theta}) \\
& \dot{y} \rightarrow \dot{y}-\frac{m r}{\sigma(M+m)}(\cos \phi \sin \theta \dot{\phi}+\sin \phi \cos \theta \dot{\theta})
\end{aligned}
$$

and with the addition of the term

$$
\begin{aligned}
\frac{1}{2} g_{\sigma}\left(\tau(v)_{Q}, \tau(v)_{Q}\right) \\
=\frac{1}{2} \frac{m^{2} r^{2}}{\sigma(M+m)}\left\{(\cos \phi \cos \theta \dot{\phi}-\sin \phi \sin \theta \dot{\theta})^{2}\right. \\
\left.\quad+(\cos \phi \sin \theta \dot{\phi}+\sin \phi \cos \theta \dot{\theta})^{2}\right\} \\
=\frac{1}{2} \frac{m^{2} r^{2}}{\sigma(M+m)}\left(\cos ^{2} \phi \dot{\phi}^{2}+\sin ^{2} \phi \dot{\theta}^{2}\right) .
\end{aligned}
$$

Control Law: Using (3.2), the control law is

$$
\begin{aligned}
u_{x} & =-\frac{d}{d t}\left(g_{x x} \tau_{\alpha}^{x} \dot{x}^{\alpha}+g_{x y} \tau_{\alpha}^{y} \dot{y}^{\alpha}\right) \\
& =\frac{d}{d t} \frac{m r}{\sigma}(\cos \phi \cos \theta \dot{\phi}-\sin \phi \sin \theta \dot{\theta}) \\
u_{y} & =-\frac{d}{d t}\left(g_{y x} \tau_{\alpha}^{x} \dot{x}^{\alpha}+g_{y y} \tau_{\alpha}^{y} \dot{y}^{\alpha}\right) \\
& =\frac{d}{d t} \frac{m r}{\sigma}(\cos \phi \sin \theta \dot{\phi}+\sin \phi \cos \theta \dot{\theta}) .
\end{aligned}
$$

We can use the general formula (3.12) to calculate this control law with the acceleration terms eliminated. We begin by computing the matrix (3.8) and find (4.7) at the bottom of the page. We now compute successively the terms in (3.12). Consider firstly $g_{a \beta, \gamma} \dot{x}^{\beta} \dot{x}^{\gamma}$. We have

$$
\begin{aligned}
& g_{x \beta, \gamma} \dot{x}^{\beta} \dot{x}^{\gamma} \\
& \quad=-m r\left(\sin \phi \cos \theta\left(\dot{\theta}^{2}+\dot{\phi}^{2}\right)+2 \cos \phi \sin \theta \dot{\theta} \dot{\phi}\right) \\
& g_{y, \beta, \gamma} \operatorname{dot} x^{\beta} \dot{x}^{\gamma} \\
& \quad=m r\left(-\sin \phi \sin \theta\left(\dot{\theta}^{2}+\dot{\phi}^{2}\right)+2 \cos \phi \cos \theta \dot{\theta} \dot{\phi}\right) .
\end{aligned}
$$

We next consider the expression

$$
B_{\alpha} \equiv\left[g_{\alpha \beta, \gamma}-\frac{1}{2} g_{\beta \gamma, \alpha}-\left(1-\frac{1}{\sigma}\right) g_{\alpha d} g^{d a} g_{\beta a, \gamma}\right] \dot{x}^{\beta} \dot{x}^{\gamma} .
$$

For $\alpha=\theta$ this yields the expression

$$
\begin{aligned}
B_{\theta} \equiv & 2 m r^{2} \sin \phi \cos \phi \dot{\theta} \dot{\phi} \\
& -\left(1-\frac{1}{\sigma}\right) \frac{2 m^{2} r^{2}}{M+m} \sin \phi \cos \phi \dot{\theta} \dot{\phi}
\end{aligned}
$$

$$
\left[\begin{array}{c}
\dot{\theta} \\
\dot{\phi} \\
\dot{x} \\
\dot{y}
\end{array}\right]^{T}\left[\begin{array}{cccc}
m r^{2} \sin ^{2} \phi & 0 & -m r \sin \phi \sin \theta & m r \sin \phi \cos \theta \\
0 & m r^{2} & m r \cos \phi \cos \theta & m r \cos \phi \sin \theta \\
-m r \sin \phi \sin \theta & m r \cos \phi \cos \theta & m+M & 0 \\
m r \sin \phi \cos \theta & m r \cos \phi \sin \theta & 0 & m+M
\end{array}\right]\left[\begin{array}{c}
\dot{\theta} \\
\dot{\phi} \\
\dot{x} \\
\dot{y}
\end{array}\right]
$$

$$
A_{\alpha \beta}=\left[\begin{array}{cc}
m r^{2} \sin ^{2} \phi-\left(1-\frac{1}{\sigma}\right) \frac{m^{2} r^{2}}{M+m} \sin ^{2} \phi & 0 \\
0 & m r^{2}-\left(1-\frac{1}{\sigma}\right) \frac{m^{2} r^{2}}{M+m} \cos ^{2} \phi
\end{array}\right]
$$


and for $\alpha=\phi$ we obtain

$$
\begin{aligned}
B_{\phi} \equiv & -m r^{2} \sin \phi \cos \phi \dot{\theta}^{2}+\left(1-\frac{1}{\sigma}\right) \frac{m^{2} r^{2}}{M+m} \\
& \times \sin \phi \cos \phi\left(\dot{\theta}^{2}+\dot{\phi}^{2}\right) .
\end{aligned}
$$

Finally, we consider the expression $g_{a} \delta A^{\delta \alpha} V_{, \alpha}$. We have

$$
\begin{aligned}
& g_{x \delta} A^{\delta \alpha} V_{, \alpha}=-\frac{m^{2} r^{2} g}{A_{\phi \phi}} \cos \phi \sin \phi \cos \theta \\
& g_{y \delta} A^{\delta \alpha} V_{, \alpha}=-\frac{m^{2} r^{2} g}{A_{\phi \phi}} \cos \phi \sin \phi \sin \theta
\end{aligned}
$$

Using (4.7)-(4.12) we obtain the complete control law

$$
\begin{aligned}
& u_{x}=\frac{-m^{2} r^{3}}{\sigma A_{\phi \phi}} \sin \phi \cos \theta\left(\sin ^{2} \phi \dot{\theta}^{2}+\dot{\phi}^{2}-\frac{g}{r} \cos \phi\right) \\
& u_{y}=\frac{-m^{2} r^{3}}{\sigma A_{\phi \phi}} \sin \phi \sin \theta\left(\sin ^{2} \phi \dot{\theta}^{2}+\dot{\phi}^{2}-\frac{g}{r} \cos \phi\right)
\end{aligned}
$$

Stabilization: We now use Theorem 3.4 to analyze stability of the pendulum about its upright state, modulo motion in the plane. We have

$$
\begin{aligned}
\frac{1}{2} A_{\alpha \beta} \dot{x}^{\alpha} \dot{x}^{\beta} & +V \\
=\frac{1}{2} m r^{2} & \left\{\left(1+\frac{m}{M+m}\left(\frac{1-\sigma}{\sigma}\right) \cos ^{2} \phi\right) \dot{\phi}^{2}\right. \\
& \left.+\left(1+\frac{m}{M+m}\left(\frac{1-\sigma}{\sigma}\right) \sin ^{2} \phi\right) \dot{\theta}^{2}\right\} \\
& -m g r(1-\cos \phi) .
\end{aligned}
$$

Note that this is independent of $\theta$, reflecting an additional rotational symmetry in this case. Consider the relative equilibrium $\phi=\dot{\phi}=0$. Then, modulo the $\theta$ directions, the second variation of (4.15) is given by the matrix as shown at the bottom of the page where $\dot{\theta}=\mu$.

Setting $\sigma=-1 / \kappa$ as in the planar pendulum we thus have the following criteria for stability.

- If $\mu=0$, we require

$$
\left(1-\frac{m}{M+m}(\kappa+1)\right)<0
$$

i.e., $\kappa>M / m$.

- If $\mu>0$, we need

and

$$
\left(1-\frac{m}{M+m}(\kappa+1)\right)
$$

$$
m r^{2}\left(-\frac{m}{M+m}(\kappa+1)\right) \mu^{2}-m g r
$$

to have the same sign.
Remark: In this analysis, the extra $S^{1}$ symmetry is simply ignored, even though it does lead to an additional conservation law. This is because the straight upright solution is a relative equilibrium for just the translation group. If we were trying to stabilize an inverted uniformly rotating equilibrium then we would have to take this extra $S^{1}$ symmetry into account. In principle this is straightforward.

\section{AsYMPtotic StABILIZATION}

We now undertake to modify our stabilizing control laws to obtain asymptotic stabilization. This is done, roughly speaking, by using the controls to simulate dissipation. However, this is not entirely straightforward, primarily because the energy for the controlled system we consider has a maximum at the relative equilibrium in the $x, \dot{x}$ variables, but the $\theta$ direction is still a symmetry direction as discussed above. We will use the feedback controls to give active dissipation. (Note that one can easily adjust the theory below if the controlled system has a minimum at the relative equilibrium in the $x, \dot{x}$ variables.)

\section{A. Theory}

To achieve our goal of converting a relative equilibrium that has been stabilized by the method of controlled Lagrangians into an asymptotically stable one, we proceed in the following step-by-step manner. The general technique here is, at least philosophically, closely related to that of [1]. Related ideas on asymptotic stabilization may be found in [15] for example.

1) Start with the original controlled system

$$
\begin{aligned}
\frac{d}{d t} \frac{\partial L}{\partial \dot{x}^{\alpha}}-\frac{\partial L}{\partial x^{\alpha}} & =0 \\
\frac{d}{d t} \frac{\partial L}{\partial \dot{\theta}^{a}} & =u_{a} .
\end{aligned}
$$

2) Choose a relative equilibrium, say $x=x_{e}$ for $L$ that is to be stabilized. Let it have momentum $\mu$ and velocity vector $\xi$, so that $\xi^{a}=g^{a b} \mu_{b}$.

3) Break the control into a conservative and dissipative piece: $u_{a}=u_{a}^{\text {cons }}+u_{a}^{\text {diss }}$, each piece of which will be defined as we proceed.

4) Assume that the hypotheses of the First Matching Theorem (2.2) hold and choose $u_{a}^{\text {cons }}=-(d / d t)\left(g_{a b} \tau_{\alpha}^{b} \dot{x}^{\alpha}\right)$ according to the controlled Lagrangian stabilizing techniques developed so far.

5) Rewrite (5.1) with $u_{a}=u_{a}^{\text {cons }}+u_{a}^{\text {diss }}$ in terms of the controlled Lagrangian. In fact, one has the following.

Proposition 5.1: Using the procedure just outlined, (5.1) is equivalent to

$$
\begin{aligned}
\frac{d}{d t} \frac{\partial L_{\tau, \sigma}}{\partial \dot{x}^{\alpha}}-\frac{\partial L_{\tau, \sigma}}{\partial x^{\alpha}} & =\tau_{\alpha}^{b} u_{b}^{\text {diss }} \\
\frac{d}{d t} \frac{\partial L_{\tau, \sigma}}{\partial \dot{\theta}^{a}} & =u_{a}^{\text {diss }} .
\end{aligned}
$$

$$
\left[\begin{array}{cc}
m r^{2}\left(1+\frac{m}{M+m}\left(\frac{1-\sigma}{\sigma}\right)\right) & 0 \\
0 & m r^{2}\left(\frac{m}{M+m}\left(\frac{1-\sigma}{\sigma}\right)\right) \mu^{2}-m g r
\end{array}\right]
$$


The explicit formula for the control law with accelerations eliminated is given by

$$
u_{a}=(\text { rhs of (3.10) })+g_{a b} \tau_{\delta}^{b} A^{\delta \alpha} g_{\alpha d} g^{d c} u_{c}^{\text {diss }}+u_{a}^{\text {diss }} .
$$

Proof: The strategy is to repeat the derivation of the matching equations. First of all, notice that the second equation in the set (5.2) can be equivalently written as

$$
\frac{d}{d t}\left(\tilde{J}_{a}\right)=u_{a}^{\text {diss }}
$$

Subtracting this from the equation

$$
\frac{d}{d t} J_{a}=u_{a}^{\mathrm{cons}}+u_{a}^{\mathrm{diss}}
$$

shows that the second equation in (5.2) is equivalent to the equation

$$
u_{a}^{\mathrm{cons}}=\frac{d}{d t}\left(J_{a}-\tilde{J}_{a}\right)
$$

which, as in Section III-A determines the control law $u_{a}^{\text {cons }}$. The explicit formula (5.3) is derived following the same steps as in Section III-A but with the zero on the right-hand side of (3.4) replaced with $u_{a}^{\text {diss }}$.

Now one goes through the computation of the Euler-Lagrange expression $\mathcal{E}\left(L_{\tau, \sigma}\right)$ given in the proof of the First Matching Theorem. The critical thing is that in (2.19) one does not replace the time derivative of $\tilde{J}_{b}$ with zero, but rather with $u_{b}^{\text {diss }}$. All other terms disappear, as in the proof of the First Matching Theorem, leaving the first equation in (5.2) as stated.

The Controlled Liapunov Function: The next step in the procedure is

6) Find a candidate Liapunov function (to be called $\mathcal{V}_{\mu}$ ).

Of course, it is natural to make use of the function that we employed to give us stability. However, as we shall see, this function must be modified in a nontrivial way.

Let $E_{\tau, \sigma}$ be the energy function for the controlled Lagrangian $L_{\tau, \sigma}$ and let $E_{\tau, \sigma}^{\xi}$ be the controlled augmented energy function defined by

$$
E_{\tau, \sigma}^{\xi}=E_{\tau, \sigma}-\tilde{J}_{b} \xi^{b} .
$$

The augmented energy has the property that its restriction to a level set of the momentum gives the energy function

$$
E_{\mu}:=\frac{1}{2} A_{\alpha \beta} \dot{x}^{\alpha} \dot{x}^{\beta}+V_{\mu}
$$

used in the stability test (this is seen by a direct calculation or by using facts from the energy-momentum method or Routh reduction). Here, $\mu$ is the value of the momentum at equilibrium and $V_{\mu}$ is the amended potential [see (3.19)]. Note that at equilibrium, the value of $\tilde{J}_{a}$ is the same as that of $J_{a}$; i.e., $\tilde{\mu}=\mu$.

A direct calculation using (5.2) shows that

$$
\frac{d}{d t} E_{\tau, \sigma}=u_{a}^{\text {diss }}\left(\dot{\theta}^{a}+\tau_{\alpha}^{a} \dot{x}^{\alpha}\right)
$$

and therefore that

$$
\frac{d}{d t} E_{\tau, \sigma}^{\xi}=u_{a}^{\mathrm{diss}}\left(\left[\dot{\theta}^{a}-\xi^{a}\right]+\tau_{\alpha}^{a} \dot{x}^{\alpha}\right) .
$$

This can be rewritten in the following way:

$$
\frac{d}{d t} E_{\tau, \sigma}^{\xi}=g^{a b} u_{a}^{\mathrm{diss}}\left(\tilde{J}_{b}-\mu_{b}-g_{\alpha b} \dot{x}^{\alpha}\right) .
$$

We also note that

$$
\frac{d}{d t} E_{\mu}=-g^{a b} u_{a}^{\mathrm{diss}} g_{\alpha b} \dot{x}^{\alpha} .
$$

Here is a crucial point: as we have seen in the examples, the function $E_{\tau, \sigma}^{\xi}$ has a maximum in the variable $x$ at the relative equilibrium in question. However, it will typically have a minimum in the variable $\dot{\theta}$. To create a function with a maximum in the variables $\left(x^{\alpha}, \dot{x}^{\alpha}, \dot{\theta}^{a}-\xi^{a}\right)$ at the equilibrium, we form the following controlled Liapunov function:

$$
\mathcal{V}_{\mu}=E_{\mu}-\frac{1}{2} \lambda g^{a b}\left(\tilde{J}_{a}-\mu_{a}\right)\left(\tilde{J}_{b}-\mu_{b}\right)
$$

where $\lambda$ is a positive constant.

The Time Derivative of $\mathcal{V}_{\mu}$ : The next step is:

7) Compute the time derivative of $\mathcal{V}_{\mu}$ and examine its definiteness.

We will compute the time derivative of $\mathcal{V}_{\mu}$ under the simplifying hypothesis that $g^{a b}$ is independent of $x^{\alpha}$. One gets

$$
\begin{aligned}
\frac{d}{d t} \mathcal{V}_{\mu} & =-g^{a b} u_{a}^{\text {diss }} g_{\alpha b} \dot{x}^{\alpha}-\lambda g^{a b} u_{a}^{\text {diss }}\left(\tilde{J}_{b}-\mu_{b}\right) \\
& =-g^{a b} u_{a}^{\text {diss }}\left(\lambda\left[\tilde{J}_{b}-\mu_{b}\right]+g_{\alpha b} \dot{x}^{\alpha}\right) .
\end{aligned}
$$

This leads us to the last step.

8) Define the dissipative control law

$$
u_{a}^{\text {diss }}=-c_{a}^{b}\left(\lambda\left[\tilde{J}_{b}-\mu_{b}\right]+g_{\alpha b} \dot{x}^{\alpha}\right)
$$

where $c_{a}^{b}$ is a positive definite, possibly $x^{\alpha}$ dependent, control gain matrix.

Note that the dissipative control law depends linearly on $\dot{\theta}^{a}$ since $\tilde{J}_{a}$ does.

We are now ready to formulate our main result on asymptotic stabilization. We will make the following assumption that is essentially a condition on the nontriviality of the coupling terms $g_{\alpha a}$ between the control variables $\theta^{a}$ and the internal variables $x^{\alpha}$.

AS. Along no trajectory other than relative equilibria of the original uncontrolled Euler-Lagrange equations for $L$ is $g_{\alpha a} \dot{x}^{\alpha}$ a constant.

Theorem 5.2 (Asymptotic Stabilization): Assume that the hypotheses of the Stabilization Theorem 3.4 as well as the Simplified Matching Assumptions hold. Assume that the relative equilibrium $x_{e}, \dot{x}_{e}=0$ is a maximum of $E_{\mu}$ given by (3.20). In addition, assume condition $\mathbf{A S}$ and that the dissipative control law is chosen as in (5.7). Then the given relative equilibrium is asymptotically stable modulo the action of the group.

Proof: We have organized things so that the time derivative of $\mathcal{V}_{\mu}$ is everywhere nonnegative and it vanishes on the set $\mathcal{M}$ defined by

$$
u_{a}^{\text {diss }}=-c_{a}^{b}\left(\lambda\left[\tilde{J}_{b}-\mu_{b}\right]+g_{\alpha b} \dot{x}^{\alpha}\right)=0 .
$$

The Invariance Principle (see [4], [26], and [24]) shows that all trajectories tend to the subset of $\mathcal{M}$ that is dynamically invariant. 

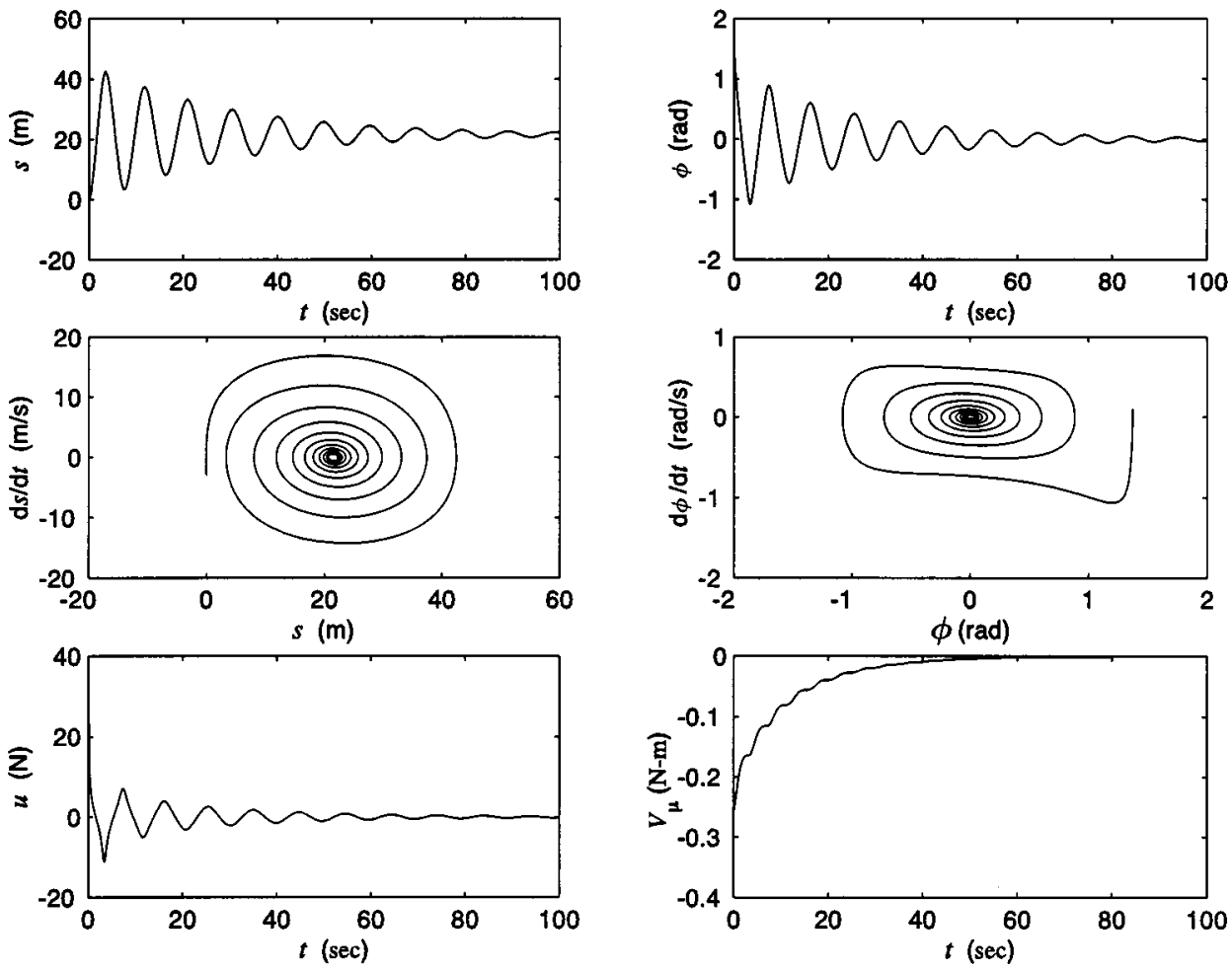

Fig. 3. Asymptotic stabilization of a pendulum starting from a nearly horizontal position.

We claim that the dynamically invariant subset of $\mathcal{M}$ consists only of equilibria (note that this set is group invariant). Suppose there is an invariant trajectory in $\mathcal{M}$. Then the time derivative of the above expression along such a trajectory is

$$
-c_{a}^{b}\left(\lambda u_{b}^{\text {diss }}+\frac{d}{d t}\left(g_{\alpha b} \dot{x}^{\alpha}\right)\right)=0
$$

and since we have a trajectory in $\mathcal{M}$, this implies $g_{\alpha a} \dot{x}^{\alpha}$ is a constant along these trajectories. Thus, $\tilde{J}_{a}$ is also constant. Under the simplified matching hypotheses, $\tilde{J}_{a}$ and $J_{a}$ differ by terms that are constant along the trajectory, so the conservative control is also zero. (One can check this directly for the pendulum.) Thus, the trajectory is a solution of the original Euler-Lagrange equations for $L$ and so by our assumption, this curve must be an equilibrium point.

We remark that assumption AS can easily be checked in specific examples as is done below. Part of this paper examines in greater depth the theory behind conditions like AS that guarantee that the Invariance Principle holds.

\section{B. Example}

We illustrate our control law design by using it to asymptotically stabilize the inverted pendulum on a cart of Sections I-B and IV-A. The relative equilibrium of interest is $\phi=\dot{\phi}=0$ and $\dot{s}=\xi$ (where $\xi=\mu / \gamma$ and $\mu$ is the desired momentum).

The explicit control law with dissipation is calculated according to (5.3) and (5.7). For the pendulum we have that

$$
g_{a b} \tau_{\delta}^{b} A^{\delta \alpha} g_{\alpha d} g^{d c} u_{c}^{\text {diss }}+u_{a}^{\text {diss }}=\frac{\alpha-\frac{\beta^{2}}{\gamma} \cos ^{2} \phi}{\alpha-\frac{\beta^{2}}{\gamma}(1+\kappa) \cos ^{2} \phi} u^{\text {diss }}
$$

and from (5.7)

$$
\begin{aligned}
u^{\text {diss }} & =-c(\lambda(\tilde{J}-\mu)+\beta \cos \phi \dot{\phi}) \\
& =-c(\lambda(\beta \cos \phi \dot{\phi}+\gamma \dot{s}+\kappa \beta \cos \phi \dot{\phi}-\mu)+\beta \cos \phi \dot{\phi}) \\
& =-c((\lambda(\kappa+1)+1) \beta \cos \phi \dot{\phi}+\lambda \gamma(\dot{s}-\xi))
\end{aligned}
$$

where $\lambda>0$ and $c>0$ and $\mu=\gamma \xi$. Substitution into (5.3) gives (5.8), shown at the bottom of the page.

We have already shown in Section IV-A that the hypotheses of Theorem 3.4 hold with the Simplified Matching Assumptions, and the relative equilibrium is a maximum of $E_{\mu}$. Condition $\mathbf{A S}$ holds since along no trajectories other than relative equilibria of the uncontrolled system is $d / d t(\beta \cos \phi \dot{\phi})=u^{\mathrm{cons}}=$ 0 . Thus, by Theorem 5.2 the control $u$ defined by (5.8) makes the equilibrium of interest asymptotically stable.

$$
u=\frac{\kappa \beta \sin \phi\left(\alpha \dot{\phi}^{2}+\cos \phi D\right)-c\left(\alpha-\frac{\beta^{2}}{\gamma} \cos ^{2} \phi\right)((\lambda(\kappa+1)+1) \beta \cos \phi \dot{\phi}+\lambda \gamma(\dot{s}-\xi))}{\alpha-\frac{\beta^{2}}{\gamma}(1+\kappa) \cos ^{2} \phi}
$$


Simulation: We demonstrate the control law with a MATLAB simulation of the pendulum-cart system where $m=0.14 \mathrm{~kg}, M=0.44 \mathrm{~kg}$, and $l=0.215 \mathrm{~m}$. We let the desired cart velocity be $\xi=0 \mathrm{~m} / \mathrm{s}$ and choose control gains to be $\kappa=135(M / m), \lambda=0.01$, and $c=50$. Fig. 3 shows plots of pendulum angle and velocity and cart position and velocity for the system subject to our stabilizing controller with dissipation added. The pendulum starts from a nearly horizontal position $(\phi(0)=\pi / 2-0.2 \mathrm{rad})$, showing the large basin of attraction for the upright pendulum. We have even given the pendulum an initial positive (downward) velocity of $\dot{\phi}(0)=0.1 \mathrm{rad} / \mathrm{s}$. The cart's initial position is $s(0)=0 \mathrm{~m}$ and initial velocity is $\dot{s}(0)=-3 \mathrm{~m} / \mathrm{s}$. Note that the cart comes to rest as desired, but due to the large initial acceleration needed to bring the pendulum to vertical, the cart drifts far from its initial position. In Part II, we present the methodology for adding another term (that breaks symmetry) to the control law in order to drive the cart position as desired. We also address more general tracking problems.

At the bottom of Fig. 3 we have included a plot of the control law $u$ and the Liapunov function $V_{\mu}$ as functions of time. The control law has an initial peak to provide the initial large acceleration. The Liapunov function can be seen to be initially negative and to strictly increase until it reaches zero at the equilibrium.

\section{ACKNOWLEDGMENT}

The authors would like to thank J. Baillieul, F. Bullo, J. Burdick, N. Getz, D. Koditschek, P. S. Krishnaprasad, R. Murray, T. Ratiu, A. Ruina, G. Sánchez de Alvarez, and C. Woolsey for helpful comments. They would also like to thank the referees for their suggestions.

\section{REFERENCES}

[1] K. J. Åström and K. Furuta, "Swinging up a pendulum by energy control," in IFAC, vol. 13, San Francisco, 1996.

[2] D. Auckly, L. Kapitanski, and W. White, "Control of nonlinear underactuated systems," Comm. Pure. Appl. Math., vol. 53, pp. 3554-3569, 2000.

[3] J. Baillieul, "Stable average motions of mechanical systems subject to periodic forcing," Fields Inst. Comm., vol. 1, pp. 1-23, 1993.

[4] E. A. Barbashin and N. N. Krasovskii, "Stability of motion in the large," Doklady Mathematics, vol. 86, no. 3, pp. 453-456, 1952. (Translations of Proc. Russian Academy Sciences).

[5] A. M. Bloch, D. E. Chang, N. E. Leonard, and J. E. Marsden, "Controlled Lagrangians and the stabilization of mechanical systems II; Potential shaping and tracking," IEEE Trans. Automat. Contr., to be published.

[6] A. M. Bloch, P. S. Krishnaprasad, J. E. Marsden, and G. Sánchez de Alvarez, "Stabilization of rigid body dynamics by internal and external torques," Automatica, vol. 28, pp. 745-756, 1992.

[7] A. M. Bloch, N. E. Leonard, and J. E. Marsden, "Stabilization of mechanical systems using controlled Lagrangians," in Proc. 36th IEEE Conf. Decision and Control, San Diego, CA, 1997, pp. 2356-2361.

[8] — " "Matching and stabilization by the method of controlled Lagrangians," in Proc. 37th IEEE Conf. Decision and Control, Tampa, FL, 1998, pp. 1446-1451

[9] — - "Stabilization of the pendulum on a rotor arm by the method of controlled Lagrangians," in Proc. IEEE Int. Conf. Robotics Automat., Detroit, MI, 1999, pp. 500-505.

[10] - "Potential shaping and the method of controlled Lagrangians," in Proc. 38th IEEE Conf. Decision Control, Phoenix, AZ, 1999, pp. 1653-1657.
[11] _ - "Controlled Lagrangians and the stabilization of Euler-Poincaré mechanical systems," Int. J. Nonlinear Robust Contr., 1999.

[12] A. M. Bloch, J. E. Marsden, and G. Sánchez de Alvarez, "Stabilization of relative equilibria of mechanical systems with symmetry," in Current and Future Directions in Applied Mathematics, M. Alber, B. Hu, and J. Rosenthal, Eds: Birkhäuser, 1997, pp. 43-64.

[13] R. W. Brockett, "Control theory and analytical mechanics," in 1976 Ames Res. Center (NASA) Conf. Geometric Control Theory, R. Hermann and C. Martin, Eds. Brookline, MA: Math. Sci. Press, 1976, vol. 7. Lie Groups: History Frontiers and Applications.

[14] P. Crouch and A. J. van der Schaft, Variational and Hamiltonian Control Systems: Springer Verlag, 1987, vol. 10. (Lecture Notes in Control and Informations Sciences)

[15] A. L. Fradkov, "Swinging control of nonlinear oscillations," Int. J. Contr., vol. 64, pp. 1189-1202, 1996.

[16] N. H. Getz and J. E. Marsden, "Control for an autonomous bicycle," in Proc. IEEE Int. Conf. Robotics Automat., Nagoya, Japan, 1995.

[17] J. Hamberg, "General matching conditions in the theory of controlled Lagrangians," in Proc. 38th IEEE Conf. Decision Control, Phoenix, AZ, 1999, pp. 2519-2523.

[18] S. M. Jalnapurkar and J. E. Marsden, "Stabilization of relative equilibria," IEEE Trans. Automat. Contr., vol. 45, pp. 1483-1491, 2000.

[19] — "Stabilization of relative equilibria II," Reg. Chaotic Dynamics, vol. 3, pp. 161-179, 1999b.

[20] N. N. Krasovskii, Stability of Motion: Stanford Univ. Press, 1959, 1963.

[21] D. E. Koditschek, "The application of total energy as a Lyapunov function for mechanical control systems," in Dynamics and Control of Multibody Systems Brunswick, ME, 1988, pp. 131-157.

[22] D. E. Koditschek and E. Rimon, "Robot navigation functions on manifolds with boundary," Adv. Appl. Math., vol. 11, pp. 412-442, 1990.

[23] W. S. Koon and J. E. Marsden, "The Poisson reduction of nonholonomic mechanical systems," Rep. Math. Phys., vol. 42, pp. 101-134, 1998.

[24] N. N. Krasovskii, Stability of Motion: Stanford Univ. Press, 1959, 1963.

[25] P. S. Krishnaprasad, "Lie-Poisson structures, dual-spin spacecraft and asymptotic stability," Nonl. Anal. Th. Meth. Appl., vol. 9, pp. 1011-1035, 1985

[26] J. P. LaSalle and S. Lefschetz, Stability by Liapunov's Direct Method, with Applications. New York: Academic, 1961.

[27] D. R. Lewis, "Lagrangian block diagonalization," Dyn. Diff. Eqns., vol. 4, pp. 1-42, 1992.

[28] N. E. Leonard, "Stabilization of underwater vehicle dynamics with symmetry-breaking potentials," Syst. Contr. Lett., vol. 32, pp. 35-42, 1997.

[29] N. E. Leonard and J. E. Marsden, "Stability and drift of underwater vehicle dynamics: Mechanical systems with rigid motion symmetry," Physica D, vol. 105, pp. 130-162, 1997.

[30] A. D. Lewis and R. Murray, "Configuration controllability of simple mechanical control systems," SIAM J. Contr. Optimiz., vol. 35, pp. 766-790, 1997.

[31] J. E. Marsden, Lectures on Mechanics: Cambridge Univ. Press, 1992, vol. 174. (London Mathematical Society Lecture Note Series).

[32] J. E. Marsden and T. S. Ratiu, Symmetry and Mechanics., 2nd ed: Springer-Verlag, 1999, vol. 17. (Texts in Applied Mathematics).

[33] J. E. Marsden and J. Scheurle, "Lagrangian reduction and the double spherical pendulum," ZAMP, vol. 44, pp. 17-43, 1993.

[34] - "The reduced Euler-Lagrange equations," Fields Institute Comm., vol. 1, pp. 139-164, 1993 b.

[35] R. A. Ortega, R. Loria, R. Kelly, and L. Praly, "On passivity-based output feedback global stabilization of Euler-Lagrange systems," Int. J. Robust and Nonlinear Control, vol. 5, no. 4, pp. 313-325, 1995. (special issue on Control of Mechanical Systems).

[36] J. C. Simo, D. R. Lewis, and J. E. Marsden, "Stability of relative equilibria I: The reduced energy momentum method," Arch. Rat. Mech. Anal., vol. 115, pp. 15-59, 1991.

[37] A. J. Van der Schaft, "Hamiltonian dynamics with external forces and observations," Math. Syst. Theory, vol. 15, pp. 145-168, 1982.

[38] — " "Stabilization of Hamiltonian systems," Nonlinear Analysis, Theory, Methods and Appl., vol. 10, pp. 1021-1035, 1986.

[39] L. S. Wang and P. S. Krishnaprasad, "Gyroscopic control and stabilization," J. Nonlinear Sci., vol. 2, pp. 367-415, 1992.

[40] D. V. Zenkov, A. M. Bloch, and J. E. Marsden, "The energy momentum method for the stability of nonholonomic systems," Dyn. Stab. Syst., vol. 13, pp. 123-166, 1998. 


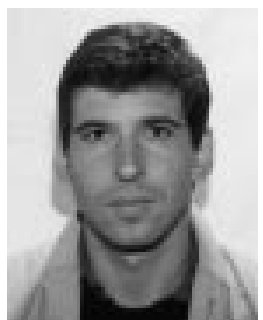

Anthony M. Bloch (S'84-M'85) is a Professor of Mathematics at the University of Michigan. He received the B.Sc. (Hons.) degree from the University of the Witwatersrand, Johannesburg in 1978, the M.S. degree from the California Institute of Technology in 1979, the M.Phil. degree from Cambridge University in 1981, and the Ph.D. degree from Harward University in 1985. His research interests are in mechanics and nonlinear control, Hamiltonian systems and integrable systems, and related areas of nonlinear dynamics. He has held visiting positions at the Mathematical Sciences Institute at Cornell University, the Mathematical Sciences Research Institute at Berkeley, and the Fields Institute in Canada, and has been a member of the Institute for Adavanced Study in Princeton. He was a T.H. Hildebrandt Assistant Professor at Michigan from 1985 to 1988 and was a faculty member at The Ohio State University before returning to Michigan in 1994. He has received a Presidential Young Investigator Award and a Guggenheim Fellowship, and he is an Associate Editor at Large for the IEEE TRANSACTIONS ON AUTOMATIC CONTROL and an Associate Editor of Mathematics of Controls, Signals and Systems.

Naomi Ehrich Leonard (S'90-M'95) received the B.S.E. degree in mechanical engineering from Princeton University in 1985 and the M.S. and Ph.D. degrees in electrical engineering from the University of Maryland in 1991 and 1994, respectively. From 1985 to 1989, she worked as an engineer in the electric power industry for MPR Associates, Inc., of Alexandria, VA.

She is currently an Associate Professor of Mechanical and Aerospace Engineering at Princeton University. She is also an associated faculty member of the Program in Applied and Computational Mathematics at Princeton. Her research interests include dynamics and control of nonlinear systems with application to mechanical systems such as autonomous underwater vehicles. Dr. Leonard is a recipient of a National Science Foundation CAREER award, 1995, an office of Naval Research Young Investigator Program Award, 1998 and an $\mathrm{Au}$ tomatica Prize Paper Award, 1999. She is co-author (with William S. Levine) of the book Using MATLAB to Analyze and Design Control Systems (AddisonWesley*Benjamin/Cummings, 1992, 2nd ed., 1995).
Jerrold E. Marsden is a Professor of Control and Dynamical Systems at the California Institute of Technology. He received the B.Sc. degree at Toronto in 1965 and the Ph.D. degree in 1968, both in applied mathematics.

He has done extensive research in the area of geometric mechanics, with applications to rigid body systems, fluid mechanics, elasticity theory, plasma physics as well as to general field theory. His primary current interests are in applied dynamics and control theory, especially how these subjects relate to mechanical systems and systems with symmetry. He was the recipient of the Norbert Wiener prize of the American Mathematical Society and the Society for Industrial and Applied Mathematics in 1990. He has been a Carnegie Fellow at Heriot-Watt University (1977), a Killam Fellow at the University of Calgary (1979), a Miller Professor at the University of California, Berkeley (1981-1982), a Humboldt Fellow in Germany (1991 and 1999), and a Fairchild Fellow at Caltech (1992). He was elected to the American Academy of Arts and Science in 1997. 